\title{
ADAPTIVE WEIGHTED LOCAL TEXTURAL FEATURES FOR ILLUMINATION, EXPRESSION AND OCCLUSION INVARIANT FACE RECOGNITION
}

\author{
Thesis \\ Submitted to \\ The School of Engineering of the \\ UNIVERSITY OF DAYTON
}

In Partial Fulfillment of the Requirements for

The Degree of

Master of Science in Electrical Engineering

By

Chen Cui

Dayton, Ohio

August, 2013

\begin{tabular}{|l|} 
UNIVERSITY of \\
DAYTON \\
DA
\end{tabular} 


\section{ADAPTIVE WEIGHTED LOCAL TEXTURAL FEATURES FOR ILLUMINATION, EXPRESSION AND OCCLUSION INVARIANT FACE RECOGNITION}

Name: Cui, Chen

APPROVED BY:

Vijayan K. Asari, Ph.D.

Advisory Committee Chairman

Professor, Electrical

and Computer Engineering
Eric J. Balster, Ph.D.

Committee Member

Professor, Electrical

and Computer Engineering

Raúl Ordóñez, Ph.D.

Committee Member

Professor, Electrical

and Computer Engineering

John G. Weber, Ph.D.

Associate Dean

School of Engineering
Tony E. Saliba, Ph.D.

Dean, School of Engineering

\&Wilke Distinguished Professor 
(C)Copyright by

Chen Cui

All rights reserved

2013 


\begin{abstract}
ADAPTIVE WEIGHTED LOCAL TEXTURAL FEATURES FOR ILLUMINATION, EXPRESSION AND OCCLUSION INVARIANT FACE RECOGNITION
\end{abstract}

Name: Cui, Chen

University of Dayton

Advisor: Vijayan K. Asari

Face recognition is one of the most promising biometric methodologies for human identification in a non-cooperative security environment. Several algorithms have been developed for extracting different facial features for face recognition. Due to the various possible challenges of data captured at different lighting conditions, viewing angles, facial expressions, and partial occlusions in natural environmental conditions, automatic facial recognition is still remaining as a difficult issue that needs to be resolved. In this thesis, we propose a novel approach to tackle some of these issues by analyzing the local textural descriptions for facial feature representation. The textural information is extracted by an enhanced local binary pattern description of all the local regions in the face, and the dimensionality of the texture image is reduced by principal component analysis performed on each local face region independently. The face feature vector is obtained by concatenating the reduced dimensional weight 
set of each module (sub-region) of the face image. The weightage of each sub-region is determined by employing the local variance estimate of the respective region which represents the significance of the region. Experiments conducted on various popular face databases show promising performance of the proposed algorithm in varying lighting, expression, and partial occlusion conditions. Research work is progressing to investigate the effectiveness of the proposed face recognition method on pose varying conditions as well. It is envisaged that a multilane approach of trained frameworks at different pose bins and an appropriate voting strategy would lead to a good recognition rate in such situation. 


\section{ACKNOWLEDGEMENTS}

This is only the beginning of chasing the academic ideals. Without the support from so many nice individuals, I would not finish this thesis at the end of my Master program.

I still remember the day when I first talked with Dr. Vijayan K. Asari. He told me about the significance and the spirit of academic research. Without his professional guidance, patient explanation, and continuous encouragement, I could hardly finish my thesis. I would like to thank all of the professors who taught me with the related courses during the two years at University of Dayton, and I sincerely appreciate Dr. Eric J. Balster and Dr. Raúl Ordóñez, who spent time commenting on my thesis and attending to my defense.

I would like to thank all the members in Vision Lab and my friends who gave me various ideas and suggestions for improving the performance of my research, as well as helping me review my thesis. I really appreciate their help, and I am very touched by their support.

With the support and inspiration of my parents, I have the chance to visit and study in United States. They lead me to the engineering world, and I will do my best to achieve the dream. 


\section{TABLE OF CONTENTS}

ABSTRACT $\ldots \ldots \ldots \ldots \ldots \ldots \ldots$ iii

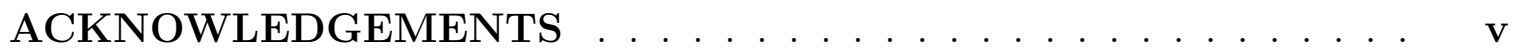

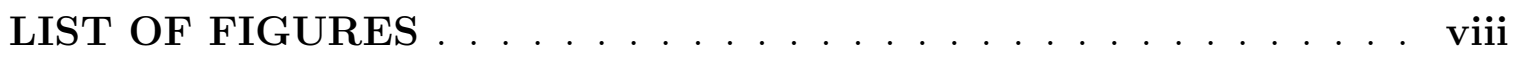

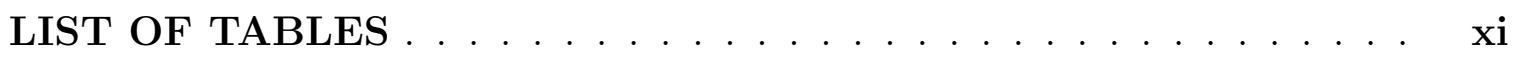

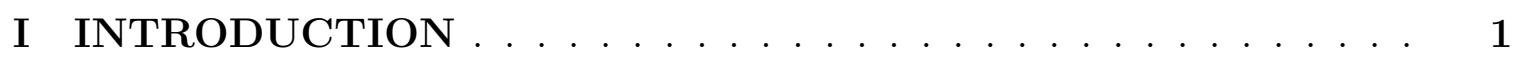

I.1 Contributions of this Thesis $\ldots \ldots \ldots \ldots \ldots$

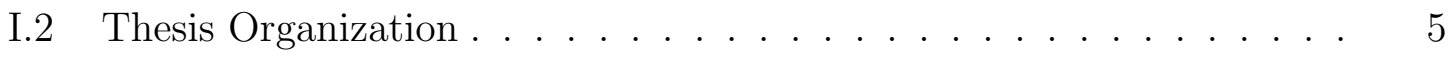

II BACKGROUND ..................... 6

II.1 Facial Recognition System . . . . . . . . . . . . . . . . 6

II.2 Face Recognition Algorithm . . . . . . . . . . . . . . . . . 10

II.3 Summary . . . . . . . . . . . . . . . . . . . . . 23

III LOCAL TEXTURAL FEATURES FOR FACE REPRESENTA-

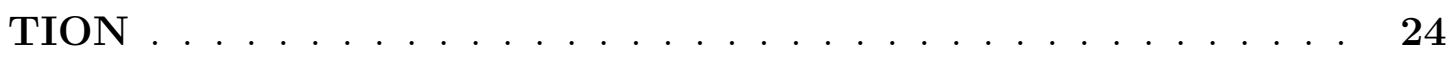

III.1 Algorithm Architecture . . . . . . . . . . . . . . . . . . . 25

III.2 Local Binary Pattern (LBP) . . . . . . . . . . . . . . 26

III.3 Local Textural Feature Weighting Strategy for Occlusion Invariant

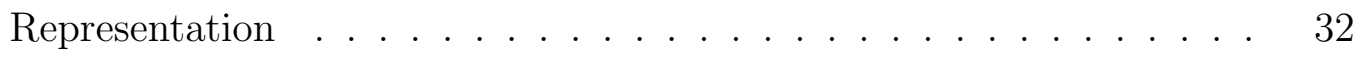


III.4 Classification . . . . . . . . . . . . . . . . . . . . . . . . 40

III.5 Summary . . . . . . . . . . . . . . . . . . . . . . . 41

IV ENHANCED LOCAL TEXTURAL REPRESENTATION FOR FACE RECOGNITION . . . . . . . . . . . . . . . . . . 42

IV.1 Enhanced LBP (ELBP) $\ldots \ldots \ldots \ldots$

IV.2 The Algorithm Steps for the enhanced LBP . . . . . . . . . . 45

IV.3 Summary . . . . . . . . . . . . . . . . . . . . . . . . 49

V EXPERIMENTAL RESULT ANALYSIS . . . . . . . . . . . 50

V.1 Experiments on Facial Expression variation Databases . . . . . 50

V.2 Experiments on Lighting Variation Database (Extended Yale B Database) 55

V.3 Experiments on Partial Occlusion issue . . . . . . . . . . 58

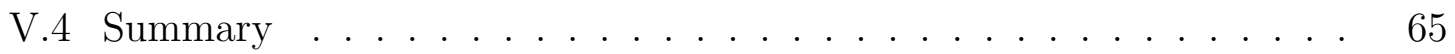

VI CONCLUSIONS AND FUTURE WORK . . . . . . . . . . . 66

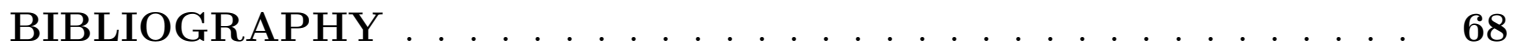




\section{LIST OF FIGURES}

I.1 Expression and lighting variations in Yale database $[2] \ldots \ldots$

I.2 Lighting variations in Extended Yale B database [3] . . . . . . . . . . 3

I.3 Expression variations in CMU AMP Facial Expression database [4] . 3

I.4 Expression variations in Japanese Female Facial Expression database [5] 3

I.5 The frame work of the proposed method . . . . . . . . . . . . . 4

II.1 A general structure for facial recognition system . . . . . . . . . . 7

II.2 Figure II.2(b) is classified as the same group as figure II.2(a) . . . . . 8

II.3 Examples in UMB-DB 3D face database [13] . . . . . . . . . . . . . . 8

II.4 Verification . . . . . . . . . . . . . . . . . 10

II.5 Identification . . . . . . . . . . . . . . . . . . . . . . . . . 11

II.6 Face detection example $[14]$. . . . . . . . . . . . . . . . . . . . . 11

II.7 LBP and CSLBP features for a neighborhood of 8 sampling points [17] 14

II.8 PCA for image dimensionality reduction . . . . . . . . . . . . . 15

II.9 Binary and Multiclass Classification . . . . . . . . . . . . . . . . 18

II.10 Single layer perceptron and multilayer perceptron architectures . . . . 20

II.11 Support Vector Machine . . . . . . . . . . . . . . . . . 23

III.1 Algorithm Architecture . . . . . . . . . . . . . . . . . 26

III.2 The basic LBP operator . . . . . . . . . . . . . . . . . . 27

III.3 Comparison with the original image and its LBP image . . . . . . . . 27 
III.4 Sampling points in $(8,1),(8,2),(16,2)$ neighborhood. The points which are not at the block center are interpolated by bilinear interpolation [16] 28

III.5 The geometric representation for bilinear interpolation [48] . . . . . . 30

III.6 Calculation strategy for uniform measure: in this sequence, there are 4 bitwise transitions from 1 to 0 or 0 to $1 \ldots \ldots 31$

III.7 The strategy for setting the label of uniform and non-uniform patterns (the first value of ulable is 0 ) . . . . . . . . . . . . . . . . 32

III.8 A face image divided into 16 modules . . . . . . . . . . . . . . . 36

III.9 The comparison between the reconstructed images from PCA and MPCA 39

IV.1 The frame work of the enhanced proposed method . . . . . . . . . . . 42

IV.2 A 3 by 3 neighborhood: $g_{1}$ to $g_{c}$ are intensity values [50] . . . . . 45

IV.3 The frame work of the proposed enhanced LBP . . . . . . . . . . 46

IV.4 Example for ELBP . . . . . . . . . . . . . . . . . . . . . . . . 47

IV.5 The comparison between LBP and ELBP images in a difficult lighting condition . . . . . . . . . . . . . . . . . . . . 49

V.1 Performance comparison with Yale database . . . . . . . . . . 51

V.2 The order for constructing the 16-bit code: starting point is $P_{3} \ldots$. . 52

V.3 The comparison with the results in [52] on Yale database . . . . . . . 52

V.4 The order for constructing the 8-bit code: starting point is $P_{3}$ (CMU AMP Facial Expression database) . . . . . . . . . . . . . . . 53

V.5 The error rates on JAFFE database . . . . . . . . . . . . 55

V.6 The comparison between general LBP and ELBP on Extended Yale B database . . . . . . . . . . . . . . . . 56 56

V.7 The order for constructing the 8-bit code: starting point is $P_{4}$ (Ex-

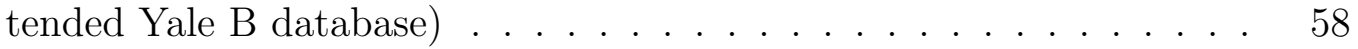


V.8 Individuals chosen from the four databases . . . . . . . . . . . . . . . 59

V.9 Occlusion examples in the four databases . . . . . . . . . . . 60

V.10 A set of examples of both eyes and eyebrows occlusions in four databases 63

V.11 The new both eyes occlusion (with eyebrows) on Yale B and CMU AMP databases ...................... . . . 64 


\section{LIST OF TABLES}

V.1 The comparison with the results in $[55,56,57]$ on CMU AMP Facial Expression database ................... . . 54

V.2 The comparison with the results in [59] on Yale B database (10 individuals) . . . . . . . . . . . . . . . . . .

V.3 The comparison with the results in [59] on Extended Yale B database (the remaining 28 individuals) . . . . . . . . . . . . . . 57

V.4 The "extreme" test on Extended Yale B database (38 individuals) . 58

V.5 The recognition results of different types of occlusions on Yale database 60

V.6 The recognition results of different types of occlusions on Yale B database 61

V.7 The recognition results of different types of occlusions on CMU AMP Facial Expression database . . . . . . . . . . . . . . . . . . . 62

V.8 The recognition results of different types of occlusions on JAFFE database 62

V.9 The recognition results of both eyes and eyebrows occlusions on four databases ........................ 64

V.10 The recognition results of the new both eyes occlusion on Yale B and CMU AMP databases . . . . . . . . . . . . . . . . . 


\section{CHAPTER I}

\section{INTRODUCTION}

Biometric verification, such as fingerprint, iris, and face recognition [1], is one of the most popular security solutions today. Using face images to identify people by machine is not a dream. Face recognition system helps to secure restricted areas by identifying and matching personal information of individuals. The technology also contributes to criminal identification. In many judicial cases, criminals are recorded by CCTV. The video frames, which contain the face of the criminal, can be used to identify. Besides video processing, this technique can be applied for evaluating identity proofs, such as ID cards, passports and other work related certificates. An effective biometric measurement saves the amount of time spent on identifying through security systems. Tracking specific people of interest could be done easily after combining tracking systems with ideal recognition algorithms. Another application is used for video gaming and other handheld game console systems. The captured face images help set up a personal profile system and save the records depending on the recognition information, therefore, there is no need for manual information entry to create different profiles.

Fingerprint and iris features are very effective for human recognition, but data collection is restricted. The cooperation of the individuals is required, and the process is strict. For instance, custom officers always let passengers press several times on the fingerprint scanner to ensure the entire fingerprint has been recorded. However, 
face information collection is not so complex. The images of individuals can be taken in the camera's field of view. Even though sometimes the face images do not contain the entire face information, there are several algorithms to obtain a high accuracy verification.

A perfect face recognition system should be able to recognize a variety of faces in different conditions, such as different poses, lighting conditions, partial occlusions, noisy data, different facial expressions, age variations, etc [1]. It is easy to train a system to recognize faces in perfect conditions; however, these intrusions make it much harder for the system to get a high recognition accuracy. Faces with different expressions of the same individual may be misclassified by the recognition system, especially with some extreme emotion expressions, such as anger and fear. Age problems occur when the same person's image taken in a different time of life. The recognition system may not realize these images belong to the same person after recognition. Also, different poses and partial occlusions may cause a similar issue. Lighting problems make the system much harder for classification. Even the human eyes can hardly recognize a person only with the face area in poor lighting conditions. The ideal face recognition system is a next to impossible.

As was mentioned before, the problems caused by different conditions can hardly be resolved in one system, so we choose three of them and find an effective solution. In this thesis, we focus on solving the facial expression variation, partial occlusions, and lighting issues. The proposed method obtains a promising result with these three conditions. Examples of the four databases, which are used for the experiments, are shown in figure 1.1, 1.2, 1.3, and 1.4. Face database of figure 1.1 contains both expression and lighting variations. Face database of figure 1.2 contains lighting issues. Face databases of figure 1.3 and 1.4 contain expression variations. 


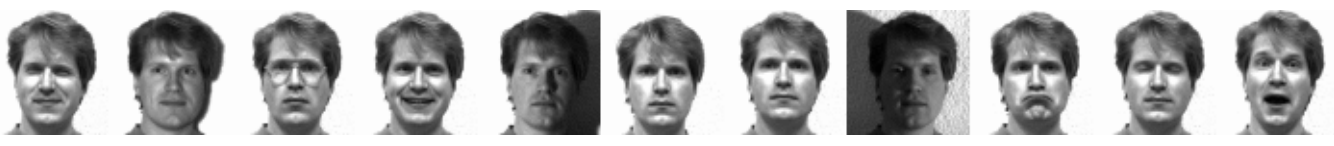

Figure I.1: Expression and lighting variations in Yale database [2]

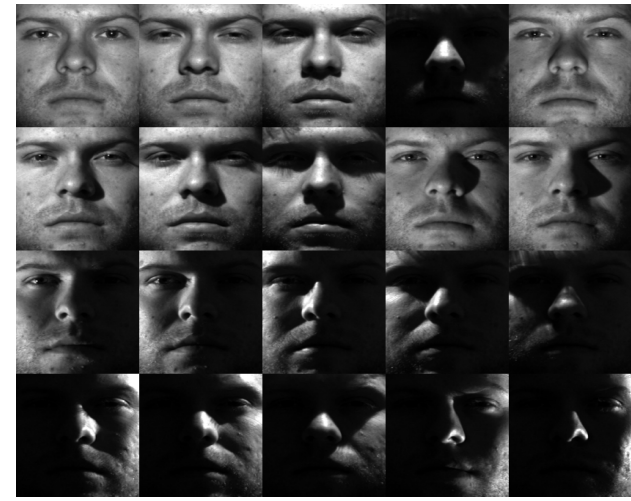

Figure I.2: Lighting variations in Extended Yale B database [3]

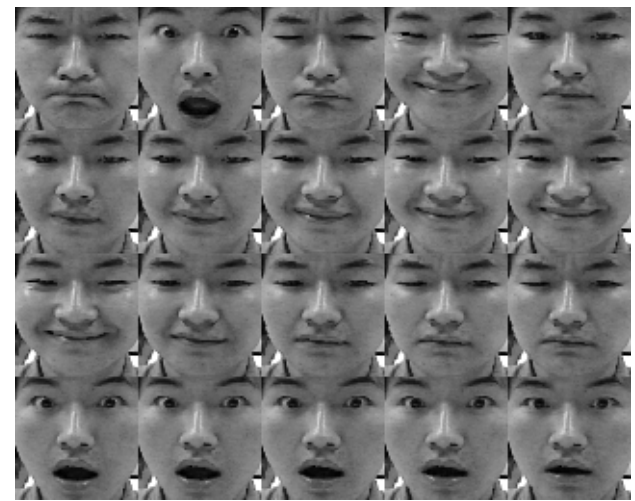

Figure I.3: Expression variations in CMU AMP Facial Expression database [4]

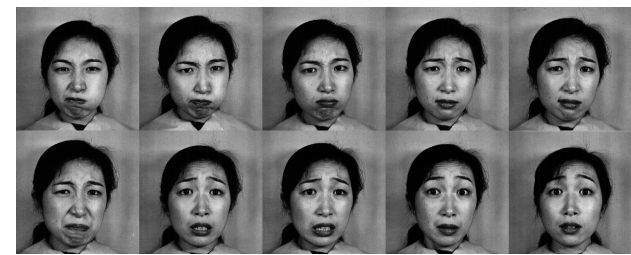

Figure I.4: Expression variations in Japanese Female Facial Expression database [5]

\section{I.1 CONTRIBUTIONS OF THIS THESIS}

Images from all the face databases have been cropped and normalized to the same size before doing any coding process. The same size images are the input patterns 
for our whole recognition system. The proposed method contributes to facial feature extraction and face recognition. It also can be used as a pre-processing stage for a face tracking system. The main contributions are shown as follows:

1. A unified framework for face recognition system.

2. A novel architecture, based on local binary pattern (LBP) and weighted modular principal component analysis (WMPCA) for face recognition.

3. An enhanced local binary pattern (ELBP) algorithm is applied for improving the performance of the recognition system.

4. The proposed method is a unique framework that can work with facial expression, partial occlusions, and lighting variation issues.

5. Tests are done with the proposed method in several face databases, and the results are compared with other methods to indicate the effectiveness.

Figure I.5 illustrates the frame work of the proposed system:

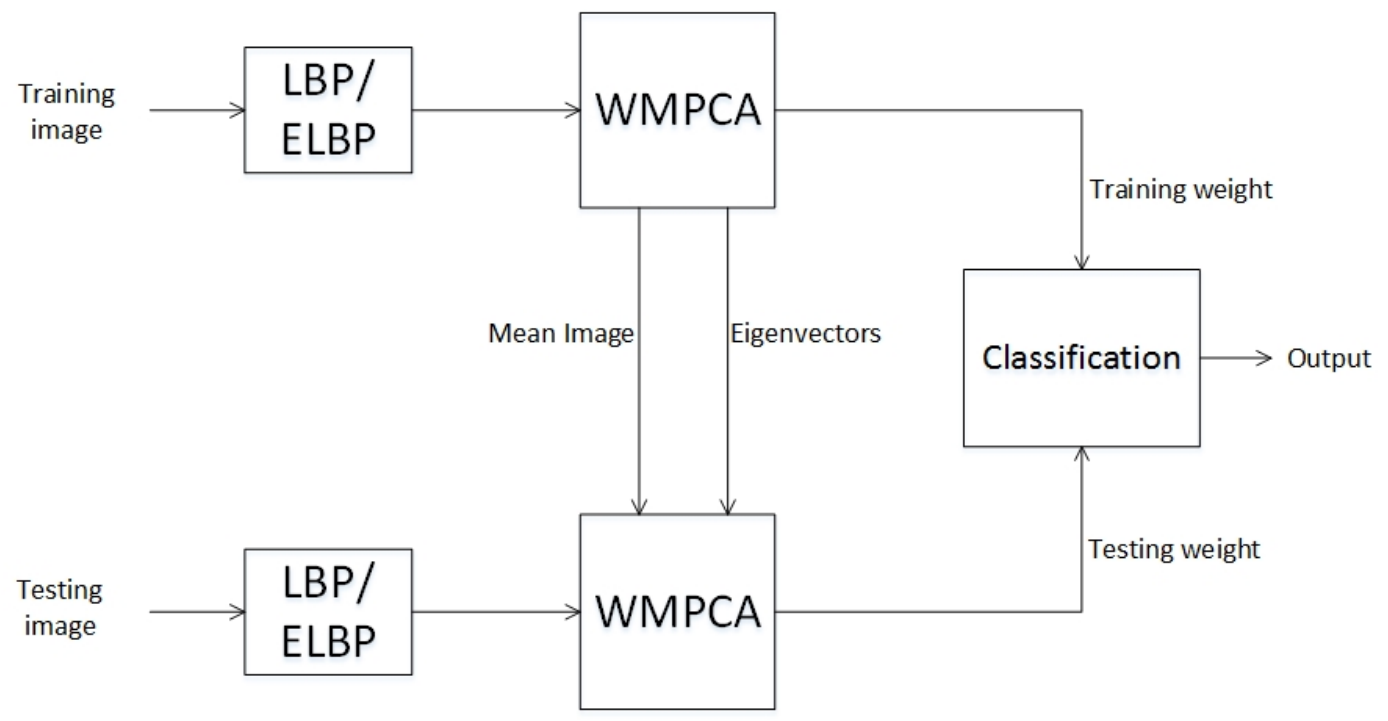

Figure I.5: The frame work of the proposed method 


\section{I.2 THESIS ORGANIZATION}

This thesis is organized as follows:

Chapter 2 gives a brief review of various related algorithms. Chapter 3 illustrates the algorithm of the proposed method: local binary pattern and weighted modular principal component analysis for face recognition. The enhanced local binary pattern and weighted modular principal component analysis for face recognition are proposed in Chapter 4. Chapter 5 shows the experimental results and the comparison with other methods. The conclusion and future work are presented in Chapter 6 . 


\section{CHAPTER II}

\section{BACKGROUND}

Face recognition of different facial expressions and lighting invariance areas have been studied for many years. There are various algorithms for facial feature extraction and classification. In this chapter, a brief history of face recognition is reviewed. First, the facial recognition system is introduced, including working theory of the facial recognition system, as well as the basic techniques and the affections. Second, some general facial recognition algorithms are presented, including the facial feature extraction and classification.

\section{II.1 FACIAL RECOGNITION SYSTEM}

A facial recognition system is a computer application for automatically identifying or verifying a person from a digital image or a video frame [6]. An ideal facial recognition system can be used for security and identification. Figure II.1 shows a general structure of facial recognition system. 

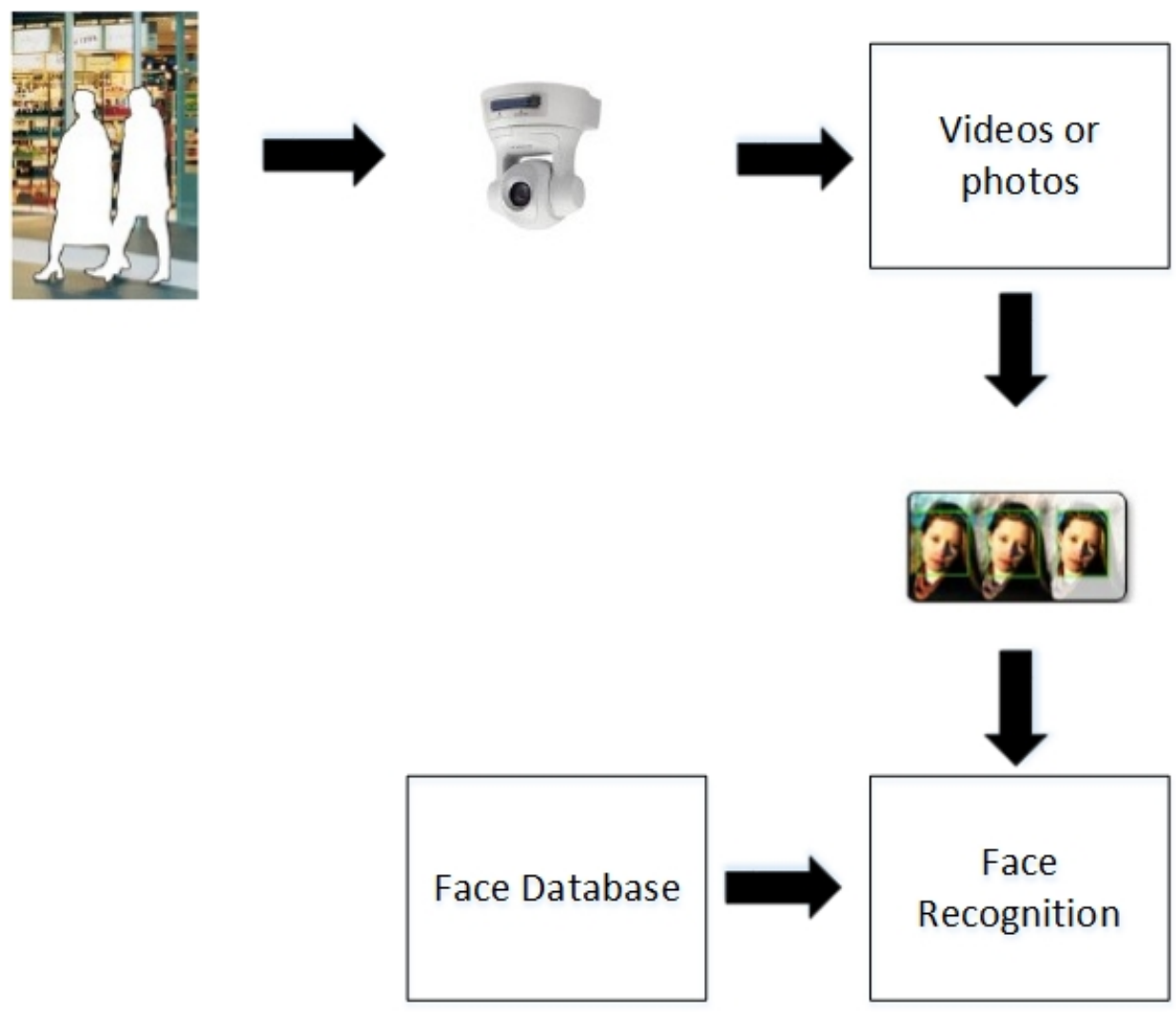

Figure II.1: A general structure for facial recognition system

\section{How to make the system work for recognition?}

One approach of system realization is to compare the selected facial features of an unknown face image with that from a large set of known faces in a database [6]. Face databases can be obtained from the intensity images, video sequences and some other sensory outputs (3D data) [7].

\section{How many techniques are used for face recognition?}

The most fundamental way for face recognition is comparing the information from probe images with the one in the recognition system (Figure II.2). The information can be separated into two parts: geometric information, which can be considered 
as a distinguishing feature; and photometric information, which can be considered as distilling an image into some values [6]. The three dimensional (3D) technology (Figure II.3) is a new tendency for face recognition. The 3D system can capture distinctive features of an individual, such as the curves of eye sockets, nose, and chin [8]. Compared with 2D facial feature extraction, the advantage of $3 \mathrm{D}$ technique is obvious: it avoids the interactions from lighting, make up and head orientation [9]. However, the cost of the 3D scanners for data collection is tremendous.

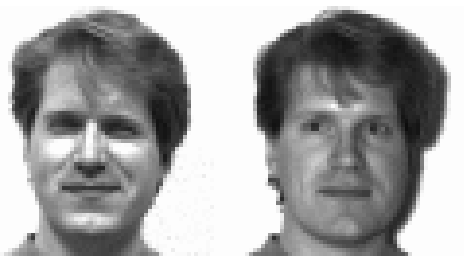

(a) Gallery image

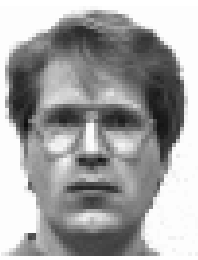

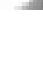

Figure II.2: Figure II.2(b) is classified as the same group as figure II.2(a)

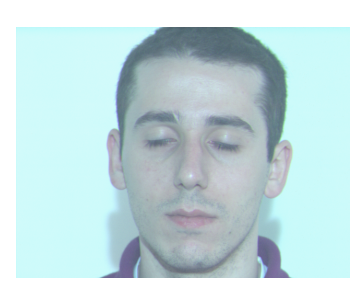

(a) Original image

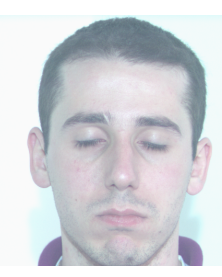

(c) Original image

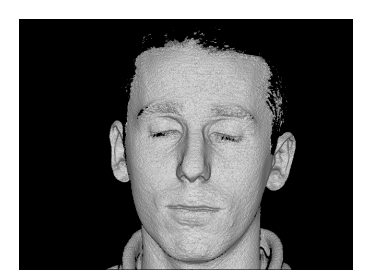

(b) 3D image

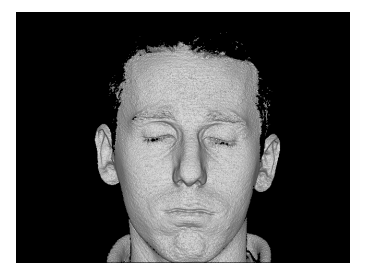

(d) 3D image

Figure II.3: Examples in UMB-DB 3D face database [13]

The technique of recognition with skin biometric information, called skin texture analysis, is also a new branch for face recognition [10]. The database is captured from 
human faces, but differs from the traditional face information, the database uses the skinprint to classify. This technique can indicate the differences between identical twins, which may not be distinguished by the traditional facial recognition system [10]. The accuracy of the results also can be increased by 20 to 25 percent $[6,8,10]$;

\section{The Comparative Study of Facial Recognition System}

As was mentioned in Chapter 1, some other biometric features can be used for individual identification, such as fingerprint and iris information. Compared with the recognition results of these biometric techniques, facial features may not have the highest accuracy. However, the advantage of facial features is arresting: there is no constraint for data collection. The face images can be taken at any places, and there is no need for the individuals' cooperation.

\section{The Pros and Cons of Facial Recognition System}

One article, written by Mike Krause, said that no positive matches were made to the criminal database in Tampa, Florida from July, 2001 to January, 2002, because male and female subjects with significant differences in age and weight [11]. This news seems to conflict with the system credited $34 \%$ reduction in crime $[6,12]$. The interesting phenomenon indicates that even though some facial recognition systems may not be necessary for criminal recognition, the acknowledgement of the system helps reduce the crimes. Even if the facial recognition system fails, it still gives the citizens a conception that the criminals can be easily recognized with the help of the system.

Although some facial recognition systems are not really helpful, the technique 
is still developing. With the help of researchers, the recognition accuracy has greatly improved in recent years.

\section{II.2 FACE RECOGNITION ALGORITHM}

Face recognition can be used in two areas: one for verification (Figure II.4), which is a one to one matching system; the other one for identification (Figure II.5), which is a one to many matching system. For the one to one system, the unknown input is compared with one known data to figure out whether the unknown individual claims to be. For the one to many system, the unknown input is compared with a bunch of known data to identify which individual it should be [7].

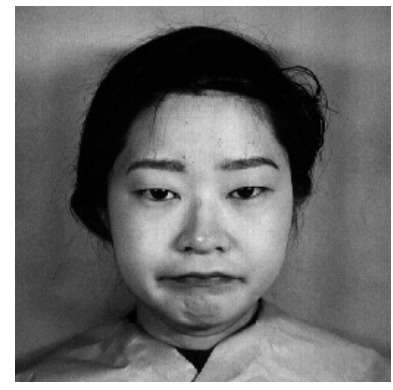

(a) Gallery image

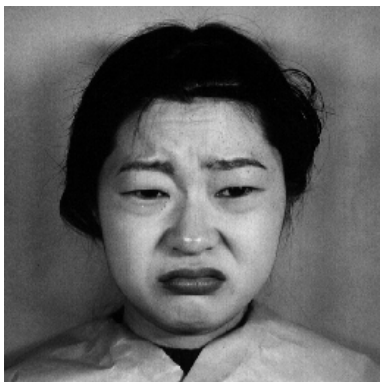

(b) Probe image

Figure II.4: Verification

In the real life, the facial recognition system is constituted by three parts: face detection (Figure II.6), facial feature extraction, and face classification. Different from the existing human face databases, most of the images captured by cameras are an entire individual, at least part of an individual. The face detection is very helpful in locating the actual position of the face part of an individual. Also, the detected position may affect the recognition results in the third part of the recognition system. After detecting the face area, the next step is facial feature extraction. The reason of applying facial feature extraction algorithms is that the original input data may 


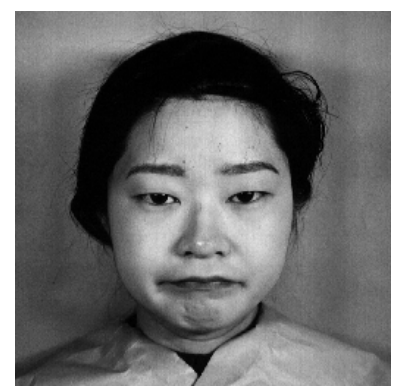

(a) Gallery image

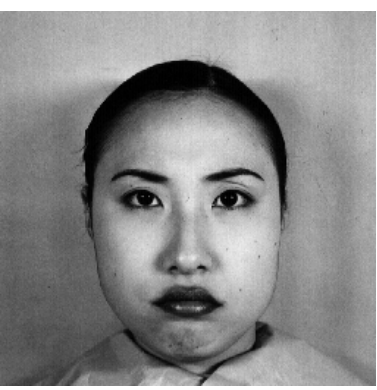

(b) Gallery image

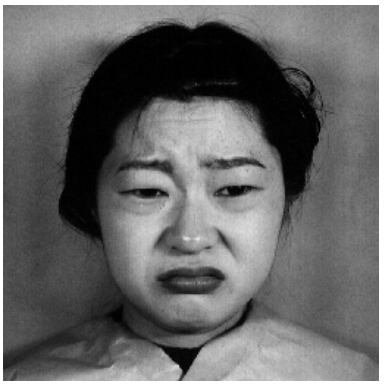

(c) Probe image

Figure II.5: Identification

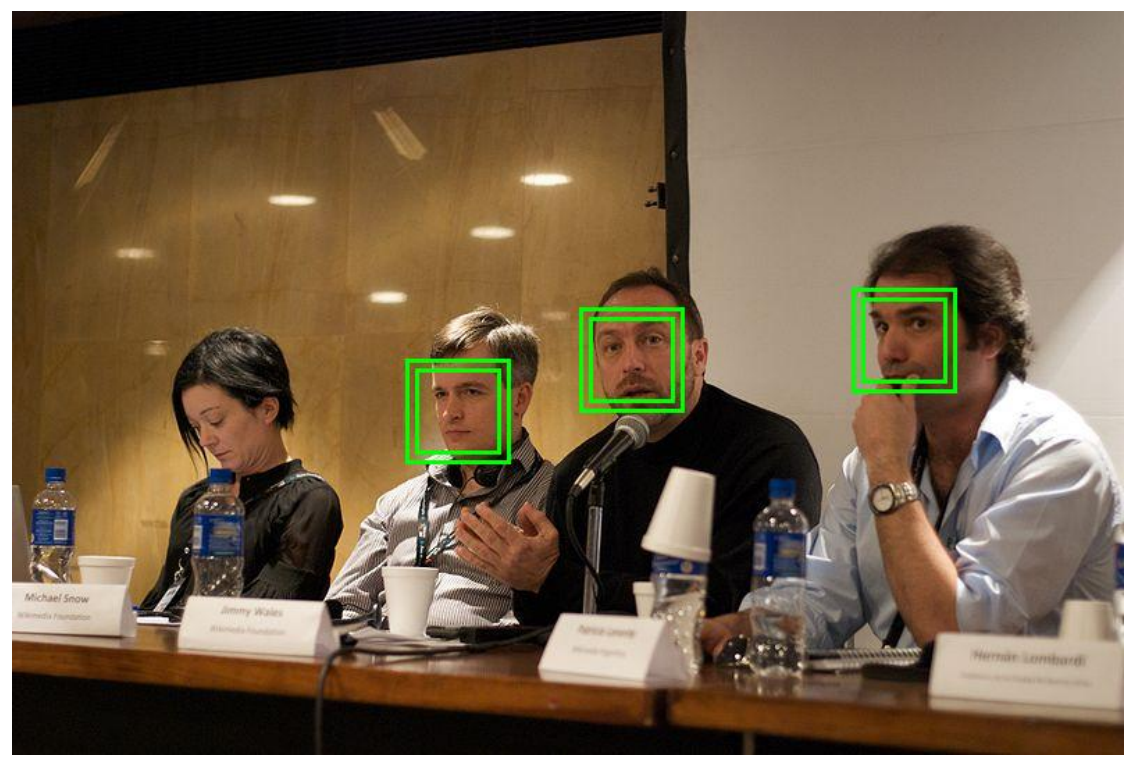

Figure II.6: Face detection example [14]

contain a large amount of undesired information, even considered as noise for the recognition system. Also, the undesired information may cause more time for system 
to go through. For the purpose of obtaining high accuracy and efficiency, facial feature extraction reduces the affection caused by the redundancies, and efficiency can be increased after omitting the information. Finally, the extracted features are considered as the inputs for the classification system. This paper concentrates on facial feature extraction and classification. More details are presented below:

\section{Facial Feature Extraction}

From the input image data, two kinds of facial features can be extracted: one is "visible" features; the other one is "invisible" features. The "visible" features can be defined as the features obtained from the original image, and there is no need to use any other techniques to process the image data, such as the color information(in RGB images). Obviously, the "invisible" feature is the opposite side, such as the eigenface [15]. There are some common methods being introduced below for facial feature extraction ("invisible" features):

\section{LBP (Local Binary Pattern) and CSLBP (Center-Symmetric Local Binary Pattern)}

The local binary pattern is a simple but efficient texture operator which recalculates the pixel labels by calculating and thresholding the differences with its neighbor pixels (Eq. II.2), and generating the binary patterns' code to represent the new label of the same pixel (Eq. II.3). The biggest advantage of LBP operator is the invariance property against monotonic gray level changes caused by illumination variations. The 
efficiency of LBP makes it work well with real-time settings.

$$
\begin{aligned}
& z_{p}=g_{p}-g_{c} \\
& s\left(z_{p}\right)= \begin{cases}1 & \text { if } z_{p} \geq 0 \\
0 & \text { if } z_{p}<0\end{cases} \\
& L B P_{P, R}\left(x_{c}, y_{c}\right)=\sum_{p=0}^{P-1} s\left(z_{p}\right) 2^{p}
\end{aligned}
$$

where $p=1,2,3, \ldots, P$ means the pixel number, $P$ is the number of sampling points (neighbor pixels). $g_{p}$ and $g_{c}$ are the intensity value of the $p$ th neighbor pixel and the center pixel respectively. $z_{p}$ is the difference between $g_{p}$ and $g_{c}$. $R$ is the radius of the neighborhood. Eq. II.2 shows the threshold rule for every neighbor pixel. $s\left(z_{p}\right)$ is the binary value of $p$ th neighbor pixel after being thresholded. $L B P_{P, R}\left(x_{c}, y_{c}\right)$ is the new label of the center pixel. $x_{c}$ and $y_{c}$ are the coordinates of the center pixel.

Instead of obtaining the binary code from comparing the value of the center pixel with that of the neighbor pixels, CSLBP obtains the new label from comparing the center symmetric pixels' values in the neighborhood. The original LBP produces 256 different new labels; however, the CSLBP produces just 16 new labels. Also, CSLBP captures better gradient information than original LBP [17]. Figure II.7 shows the difference between LBP and CSLBP. Eq. II.5 shows the final step for obtaining the new label. 


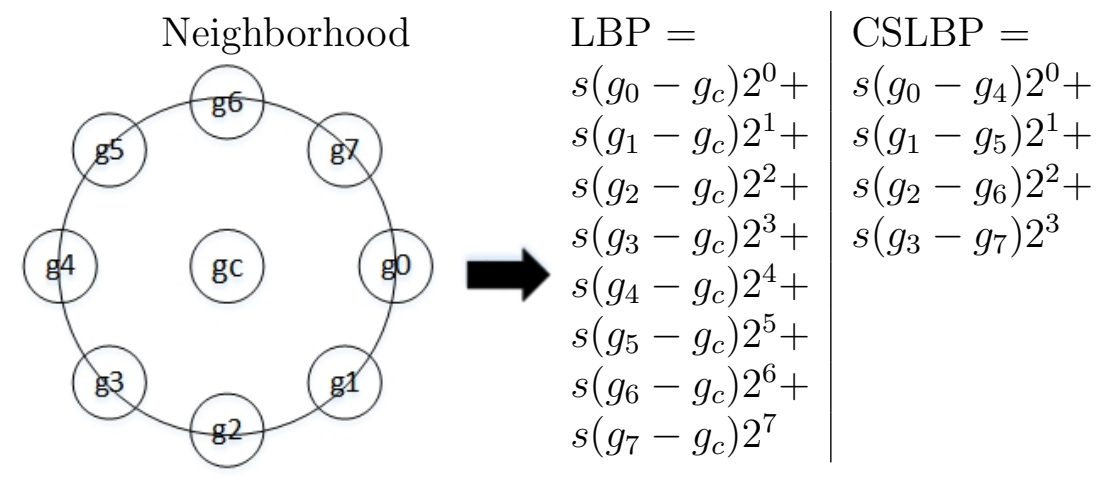

Figure II.7: LBP and CSLBP features for a neighborhood of 8 sampling points [17]

$$
\begin{aligned}
& s\left(g_{p}-g_{p+4}\right)=\left\{\begin{array}{rr}
1 & \text { if } g_{p}-g_{p+4} \geq \mathrm{T} \\
0 & \text { if } g_{p}-g_{p+4}<\mathrm{T}
\end{array}\right. \\
& \operatorname{LBP}_{P, R, T}\left(x_{c}, y_{c}\right)=\sum_{p=0}^{P / 2-1} s\left(g_{p}-g_{p+4}\right) 2^{p}
\end{aligned}
$$

where $T$ is a small threshold value. $p=0,1,2, \ldots, \frac{P}{2}-1$

\section{PCA (Principal Component Analysis)}

The principal component analysis generates the dimension reduced features instead of the original long vectors for face recognition. Faces have a similar structure (eyes, nose, mouth, etc), and the faces in the same class will group at a certain location in image space. Face images can be represented by a set of eigenvectors calculated from the covariance matrix $\mathbf{C}$ (Eq. II.6) of the training images [18].

$$
\mathbf{C}=\frac{1}{D} \sum_{d=1}^{D}\left(\mathbf{I}_{d}-\overline{\mathbf{I}}\right)\left(\mathbf{I}_{d}-\overline{\mathbf{I}}\right)^{T}
$$


where $d=1,2,3, \ldots, D, D$ is the number of training images. $\mathbf{I}_{d}$ represents the $d$ th image vector. $\overline{\mathbf{I}}$ is the mean image vector of all the training image vectors. Figure II.8 illustrates the effect of PCA.

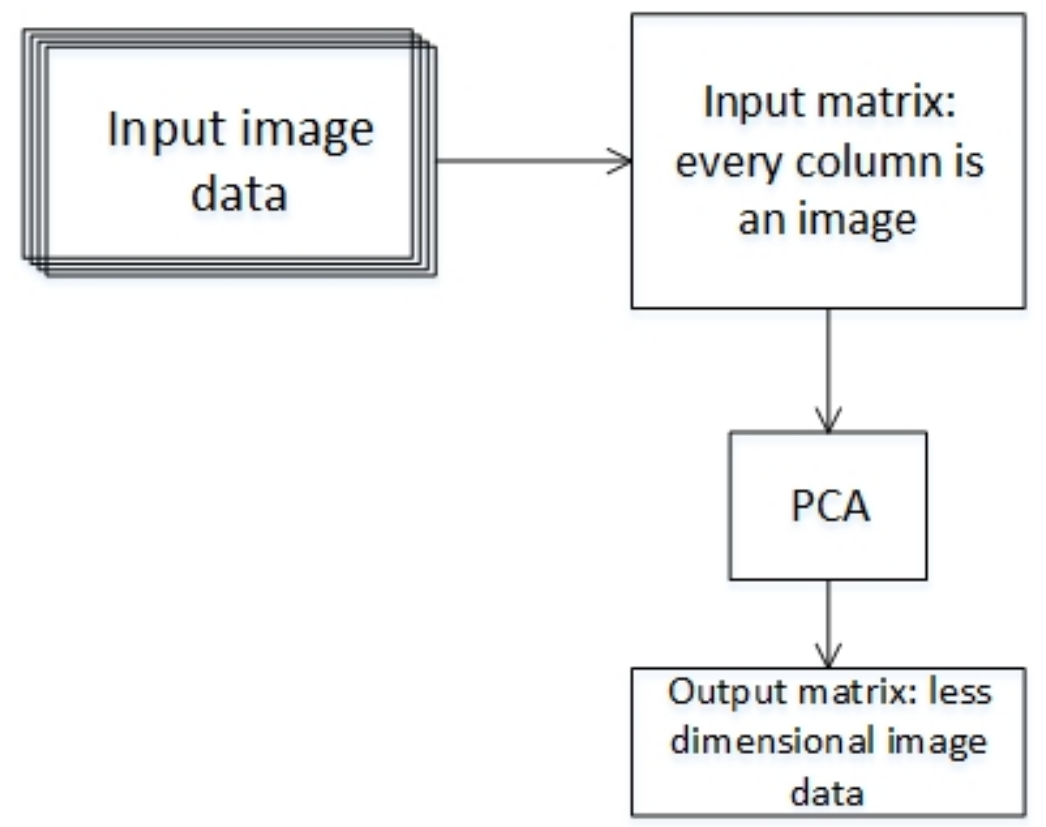

Figure II.8: PCA for image dimensionality reduction

Modular PCA (MPCA) is based on the traditional PCA. An input image is equally separated into several modules (such as 4,8,16 modules), then applying the PCA algorithm on every module. MPCA has a better result than traditional PCA [18].

Kernel PCA (KPCA) is an extension of the basic PCA by using the kernel functions $\Phi\left(\mathbf{I}_{d}\right)$ (Eq. II.7).

$$
\mathbf{C}_{k}=\frac{1}{D} \sum_{d=1}^{D}\left(\Phi\left(\mathbf{I}_{d}\right)-\Phi(\overline{\mathbf{I}})\right)\left(\Phi\left(\mathbf{I}_{d}\right)-\Phi(\overline{\mathbf{I}})\right)^{T}
$$

where $\mathbf{C}_{k}$ is the covariance matrix. Kernel functions make the linear mapping non- 
linear, and calculate the principal components in higher dimensional space. The nonlinear mapping features also give a better result than the basic linear mapping features $[19,20]$.

Weight coefficients are added to the basic PCA to produce Weighted PCA (WPCA). WPCA has been proposed in [21]. The weight coefficients are calculated by the distance between the testing and training data. Eq. II.8.a to Eq. II.8.d show the mathematical procedure for calculating the covariance matrix $\mathbf{C}_{w}$.

$$
\begin{aligned}
& \mathbf{C}_{w}=\frac{1}{D} \sum_{d=1}^{D}\left(\mathbf{I}_{d}^{\prime}-\overline{\mathbf{I}}^{\prime}\right)\left(\mathbf{I}_{d}^{\prime}-\overline{\mathbf{I}}^{\prime}\right)^{T} \\
& \mathbf{I}_{d}^{\prime}=\mathbf{w}_{d} \mathbf{I}_{d} \\
& \overline{\mathbf{I}}^{\prime}=\mathbf{w}_{d} \overline{\mathbf{I}} \\
& \mathbf{w}_{d}=\exp \left(-\frac{\max _{\text {dist }}-\operatorname{dist}\left(\mathbf{I}_{d}, \mathbf{I}_{\text {test }_{d_{\text {test }}}}\right)}{\gamma}\right)
\end{aligned}
$$

where $\mathbf{I}_{\text {test }} t_{\text {test }}$ is the $d_{\text {test }}$ th testing image. $\operatorname{dist}\left(\mathbf{I}_{d}, \mathbf{I}_{\text {test }}\right.$ dest $\left._{\text {test }}\right)$ is the distance between $\mathbf{I}_{d}$ and $\mathbf{I}_{\text {test }}$ dest $_{\text {th }}, \max _{\text {dist }}$ is the biggest distance between all the training image vectors and $\mathbf{I}_{\text {test }} t_{\text {test }}, \mathbf{w}_{d}$ is the weight matrix obtained from the training and testing patterns, $\gamma$ is a positive constant [21].

Gabor PCA (GPCA) calculates the principal components from a Gabor wavelet representation of an image. The Gabor wavelet representation is the convolution of the image with a family of Gabor kernels $\psi_{\mu, \nu}$, as $O_{\mu, \nu}=\mathbf{I}_{d} * \psi_{\mu, \nu}[22]$.

Unlike the basic PCA, 2DPCA treats the input image as a matrix rather than a vector. 2DPCA obtains a much lower dimensions of covariance matrix than basic PCA. The image covariance matrix $\mathbf{C}_{2 D}$ is calculated by the original image directly. 
Eq. II.9 shows the equation for calculating $\mathbf{C}_{2 D}$.

$$
\mathbf{C}_{2 D}=\frac{1}{D} \sum_{d=1}^{D}\left(\mathbf{I}_{2 D d}-\overline{\mathbf{I}}_{2 D}\right)\left(\mathbf{I}_{2 D d}-\overline{\mathbf{I}}_{2 D}\right)^{T}
$$

where $\mathbf{I}_{2 D d}$ is the $d$ th training image. $\overline{\mathbf{I}}_{2 D}$ is the mean image of all the training images. It should be noted that every principal component in 2DPCA is a vector, whereas the principal component in PCA is a scalar. 2DPCA gets higher efficiency and accuracy than the basic PCA [23].

\section{LDA (Linear Discriminant Analysis)}

LDA remains as much of the class discriminatory information as possible after reducing the dimensionality of the original images. Fisher's solution suggested maximizing the difference between the means, and normalized by a measure of the withinclass scatter [24]. Within-class scatter $S_{W}$ and between-class scatter $S_{B}$ are used for calculating the final Fisher criterion: $J(w)=\frac{w^{T} S_{B} w}{w^{T} S_{W} w}$, where $w^{*}=S_{W}^{-1}\left(\mu_{1}-\mu_{2}\right)$. Multiclass LDA calculates $S_{W}$ and $S_{B}$ among all the classes, and the strategy is the same as that of two classes.

\section{Face Classification}

After facial feature extraction, face classification can start. The classification rule is a procedure in which every element in a population can be assigned to one of the classes. The best performance is that every element is dispensed to the class which it should belong to. Classification can be divided into two categories: the binary classification (Figure II.9(a)) and the multiclass classification(Figure II.9(b)). 


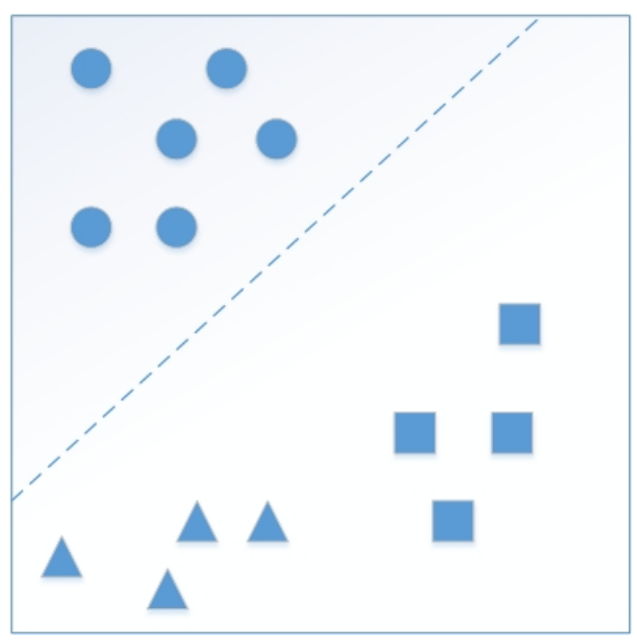

(a) Binary classification

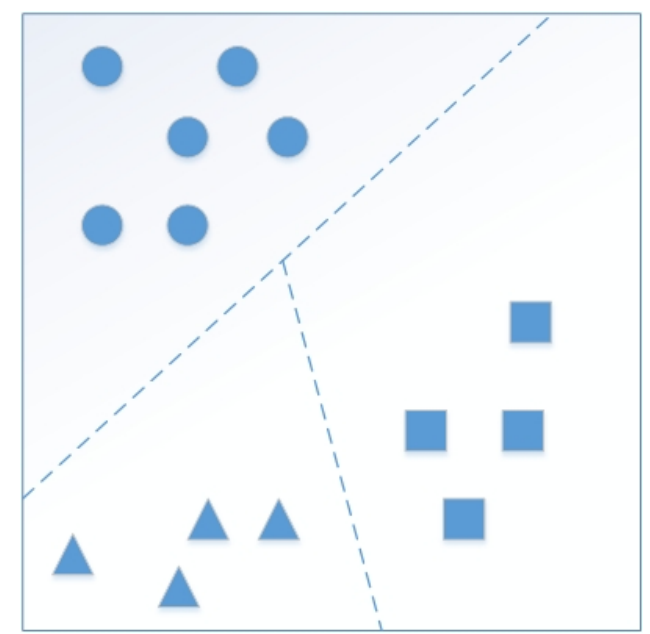

(b) Multiclass classification

Figure II.9: Binary and Multiclass Classification

In the binary classification, input data needs to be separated into only two groups; however, in multiclass classification, input data needs to be assigned into several groups [25]. Many classification algorithms belongs to binary classification, such as support vector machine. The system always combines the binary classifications together to create one multiple binary classification when there are multiple classes. For the classification algorithms, three types of learning methods can be mentioned: supervised learning, unsupervised learning, and semi-supervised learning [1]. Supervised learning is a function inferred by the training system with labeled training data. The training samples of supervised training have desired output values, and these desired output values allow the classification system to construct a mapping rule for classifying new input samples [26]. Unsupervised learning is opposite to supervised learning: the training data has no label. The hidden structure needs to be found from the unlabeled training data, and no desired output labels are used for comparing [27]. Semi-supervised learning is between supervised learning and unsupervised 
learning. The training data is constructed by a small groups of labeled data and large groups of unlabeled data [28]. This combination can improve the learning accuracy considerably. Now, some common classifier algorithms are introduced below:

\section{Euclidean Distance and Mahalanobis Distance}

In mathematics, Euclidean distance is the absolute distance between two points. The Euclidean distance between two $N$-dimensional vector $\mathbf{a}$ and $\mathbf{b}$ is calculated as Eq. II.10 [29].

$$
d_{E}=\sqrt{\sum_{i=1}^{N}\left(a_{i}-b_{i}\right)^{2}}
$$

where $d_{E}$ is the Euclidean distance between $\mathbf{a}$ and $\mathbf{b}, a_{i}$ and $b_{i}$ are the $i$ th point in $\mathbf{a}$ and $\mathbf{b}$ respectively. The testing inputs are compared with the average Euclidean distance in every class, inferring to the class which has the minimum distance. The algorithm has no limitations, and its calculation cost is low.

Mahalanobis distance was introduced by P.C. Mahalanobis in 1936 [30]. The algorithm is based on correlations between variables [31]. Consider we have a vector $\mathbf{a}=\left[a_{1}, a_{2}, a_{3}, \ldots, a_{N}\right]^{T}$, the Mahalanobis distance from a group of vectors with mean $\boldsymbol{\mu}=\left[\mu_{1}, \mu_{2}, \mu_{3}, \ldots, \mu_{N}\right]^{T}$ is defined by:

$$
d_{M}(\mathbf{a})=\sqrt{(\mathbf{a}-\boldsymbol{\mu})^{T} \mathbf{S}^{-1}(\mathbf{a}-\boldsymbol{\mu})}
$$

where $d_{M}(\mathbf{a})$ is the Mahalanobis distance. $\mathbf{S}$ is the covariance matrix of the group of vectors. The classification performance is better than Euclidean distance, but it has some requirements for the base data (the base data is the group of vectors): the quantity of observations should be larger than the dimension of every observation. 


\section{Single Layer Perceptron and Multilayer Perceptron}

Single layer perceptron (Figure II.10(a)) uses one single layer neural network to classify the input patterns; whereas, multilayer perceptron (Figure II.10(b)) uses at least two layers (one or more hidden layers and one output layer) of neural network for classification [32].

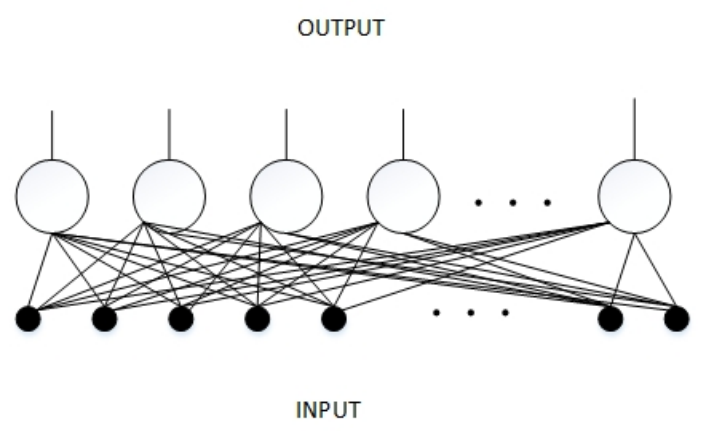

(a) Single Layer Perceptron

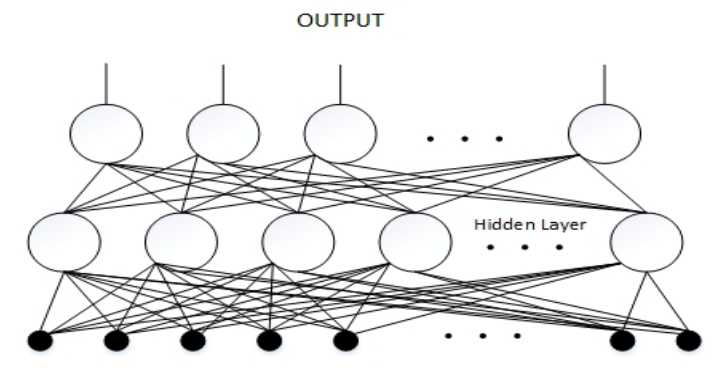

INPUT

(b) Multilayer Percenptron

Figure II.10: Single layer perceptron and multilayer perceptron architectures

Eq. II.12.a to Eq. II.12.d show the steps for calculating the outputs of single layer perceptron.

$$
\begin{aligned}
& \mathbf{h}=\mathbf{w} \mathbf{I}-\text { bias } \\
& o_{j}= \begin{cases}1 & \text { if } h_{j} \geq 0 \\
-1 & \text { if } h_{j}<0\end{cases} \\
& \mathbf{w}_{\text {new }}=\mathbf{w}+\boldsymbol{\delta} \mathbf{w} \\
& \boldsymbol{\delta} \mathbf{w}=\eta\left(\mathbf{o}^{\prime}-\mathbf{o}\right) \mathbf{I}^{T}
\end{aligned}
$$

where $\mathbf{h}$ is the output vector calculated by the system. $\mathbf{w}$ is the weight matrix. bias is a small value of bias. $\mathbf{I}$ is an image vector. $\mathbf{o}^{\prime}$ is the ideal output vector. $\mathbf{o}$ is the 
output vector after thresholding $\mathbf{h} . o_{j}$ and $h_{j}$ are the $j$ th value of $\mathbf{o}$ and $\mathbf{h} . \boldsymbol{\delta} \mathbf{w}$ is the update matrix for updating weight matrix. $\eta$ is a small positive value. Multiayer perceptron (MLP) is a feedforward artificial neural network model [33], and the first layer can be treated as a feature extraction system [34]. First, the initial random weights of two layers (hidden layer and output layer) are set respectively ( $\mathbf{v}$ and $\mathbf{w})$, and calculate the outputs of the two layers $\left(\mathbf{u}_{h}\right.$ (hidden layer) and $\mathbf{o}$ (output layer)) with the initial weights. (Eq. II.13.a to Eq. II.13.d)

$$
\begin{aligned}
& \mathbf{N e t}_{h}=\mathbf{v I}+\text { bias }_{h} \\
& \mathbf{u}_{h}=\frac{1}{1+e^{- \text {Net }_{h}}} \\
& \mathbf{N e t}_{o}=\mathbf{w u}_{h}+\text { bias }_{o} \\
& \mathbf{o}=\frac{1}{1+e^{- \text {Net }_{o}}}
\end{aligned}
$$

where $\mathbf{N e t}_{h}$ and $\mathbf{N e t}_{o}$ are two middle values of hidden layer and output layer for calculating $\mathbf{u}_{h}$ and $\mathbf{o}$. bias $s_{h}$ and bias $s_{o}$ are small values of bias for hidden layer and output layer. Then, comparing o with the desired output o', and using the difference $\boldsymbol{\delta}\left(\boldsymbol{\delta}=\mathbf{o}^{\prime}-\mathbf{o}\right)$ to update the output layer weights $\mathbf{w}$.

$$
\begin{aligned}
& \boldsymbol{\delta} \mathbf{w}=\eta \boldsymbol{\delta} \mathbf{o}(1-\mathbf{o}) \mathbf{u}_{h}^{T} \\
& \mathbf{w}_{n e w}=\mathbf{w}+\boldsymbol{\delta} \mathbf{w}
\end{aligned}
$$

where $\mathbf{w}_{\text {new }}$ is the new output weight matrix. Now, the new output weights are used 
for calculating the new hidden weight matrix $\mathbf{v}_{\text {new }}$.

$$
\begin{aligned}
& \boldsymbol{\delta} \mathbf{v}=\eta \mathbf{w}_{\text {new }} \boldsymbol{\delta} \mathbf{u}_{h}\left(1-\mathbf{u}_{h}\right) \mathbf{I}^{T} \\
& \mathbf{v}_{\text {new }}=\mathbf{v}+\boldsymbol{\delta} \mathbf{v}
\end{aligned}
$$

where $\boldsymbol{\delta} \mathbf{v}$ is the update matrix for updating $\mathbf{v}$. Repeat the procedure until the actual outputs can match the desired ones. Depending on the structure and property, MLP needs more time for classifier. The generally result is better than single layer perceptron.

\section{Support Vector Machine}

Support vector machine (SVM) (Figure II.11) makes the maximum margin boundaries between different classes of data [1]. It can create a hyperplane to divide the input patterns into two classes. $\mathbf{W} \mathbf{x}-b \geq 1$ defines the boundary of the first class, $\mathbf{W} \mathbf{x}-b \leq-1$ defines the boundary of the second class. $\mathbf{x}$ is one of the input vectors. $b$

$\frac{}{\|\mathbf{W}\|}$ decides the offset of the hyperplane from the origin along the normal vector W. Multiclass SVM can classify more than two classes input patterns. The strategy is to reduce the single multiclass problem into multiple binary classification problem. SVM can do a complex task of classification for high dimensional input patterns. 


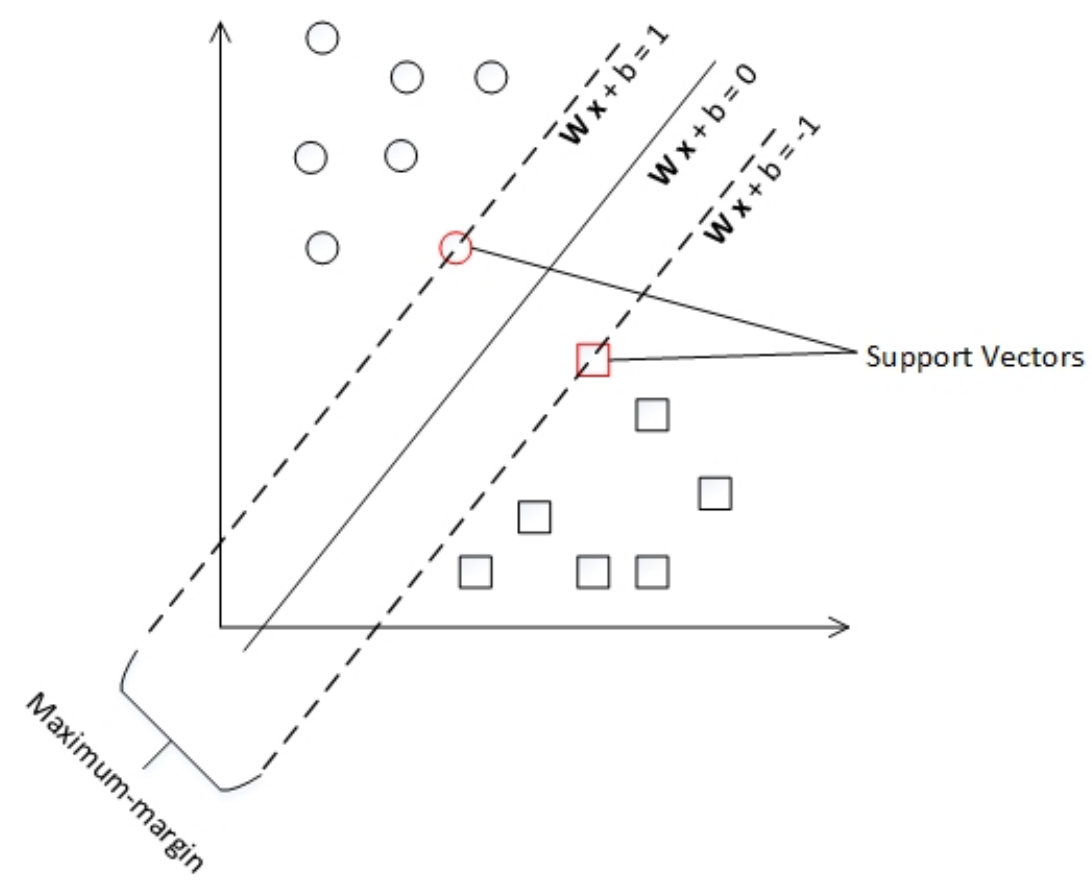

Figure II.11: Support Vector Machine

Depending on different purposes and cost problems, proper classifier algorithms are chosen for the specific systems. Sometimes, several algorithms are combined together to achieve the goal. Also, depending on the property of every algorithm, researchers need to use the fittest algorithm for classifier.

\section{II.3 SUMMARY}

In this chapter, the basic architecture of a facial recognition system is introduced. Also, some general algorithms for facial feature extraction and classification are reviewed. Even though many algorithms are listed in the two parts (facial feature extraction and face classification), the result of combining different algorithms may not be the same. One classification algorithm may obtain a good result with a unique type of facial features. Choosing the proper combination of the algorithms is crucial. 


\section{CHAPTER III}

\section{LOCAL TEXTURAL FEATURES FOR FACE REPRESENTATION}

Image texture is a set of matrices calculated by image processing algorithms to capture the perceived texture of an image. The information of the spatial arrangement of the color or intensities in an image or part of an image can be given by the image texture [35]. Texture analysis has been a topic of the intensive research since 1960s, and a wide variety of techniques for discriminating textures have been proposed [16]. Textural features have different types, such as autocorrelation based textural features, co-ocurrence matrices textural features, edge-frequency based textural features, primitive length textural features, and Law's textural features [36]. Autocorrelation based textural features depend upon the spatial size of texture primitives. The smaller the primitives are, the better texture will be given. Co-ocurrence matrices textural features are the relationships between pixels within the region by the construction of the Spatial Gray Level Dependency matrices [37]. Edge-frequency based textural features are the edge image from the original image. Primitive length textural features are represented by the number of neighbor pixels. Law's textural features are detected by applying the local masks [38].

Local Binary Pattern (LBP), proposed in 1990, is a special case of the Texture Spectrum model [39], first described in 1994 [16, 40]. LBP is a very discriminative and high efficiency texture descriptor, and shows significant improvement for solving image processing problems. Rotation-invariant LBP was developed in the late 1990s, 
and the first version was published in Pattern Recognition [16, 41]. Better algorithms, based on basic LBP, were developed in the next few years, such as Multiscale LBP and Center-Symmetric LBP. Principal Component Analysis (PCA) used for classification highlights the similarities and differences of a bunch of data. It also can be applied for data compression, because the loss of information is less after reducing the dimensions [42]. PCA was invented in 1901 by Karl Pearson [43]. PCA can be done by eigenvalue decomposition of a covariance matrix of a set of data. It is defined that the first principal component has the largest variance, and the variances of the other components are reduced in turn. Also, every component is orthogonal to the preceding components [44]. With the help of PCA, large dimensional data can be reduced to less dimensions without losing much information, thus increasing the efficiency of the whole system.

Based on the information found above, method is proposed for face recognition in face expression, partial occlusion and lighting variation face images. The proposed method uses LBP space feature for the entire face image, and obtains the first group of principal components from the feature extracted data, then applies Euclidean distance for classification. Very detailed explanation of the proposed algorithm is described in the following sections.

\section{III.1 ALGORITHM ARCHITECTURE}

The algorithm architecture of the proposed method is shown in figure III.1. The frame work includes the main algorithm - facial feature extraction by LBP, and image dimensionality reduction by weighted modular PCA (WMPCA). More details are presented in section III.2 for LBP, and section III.3 for WMPCA. 


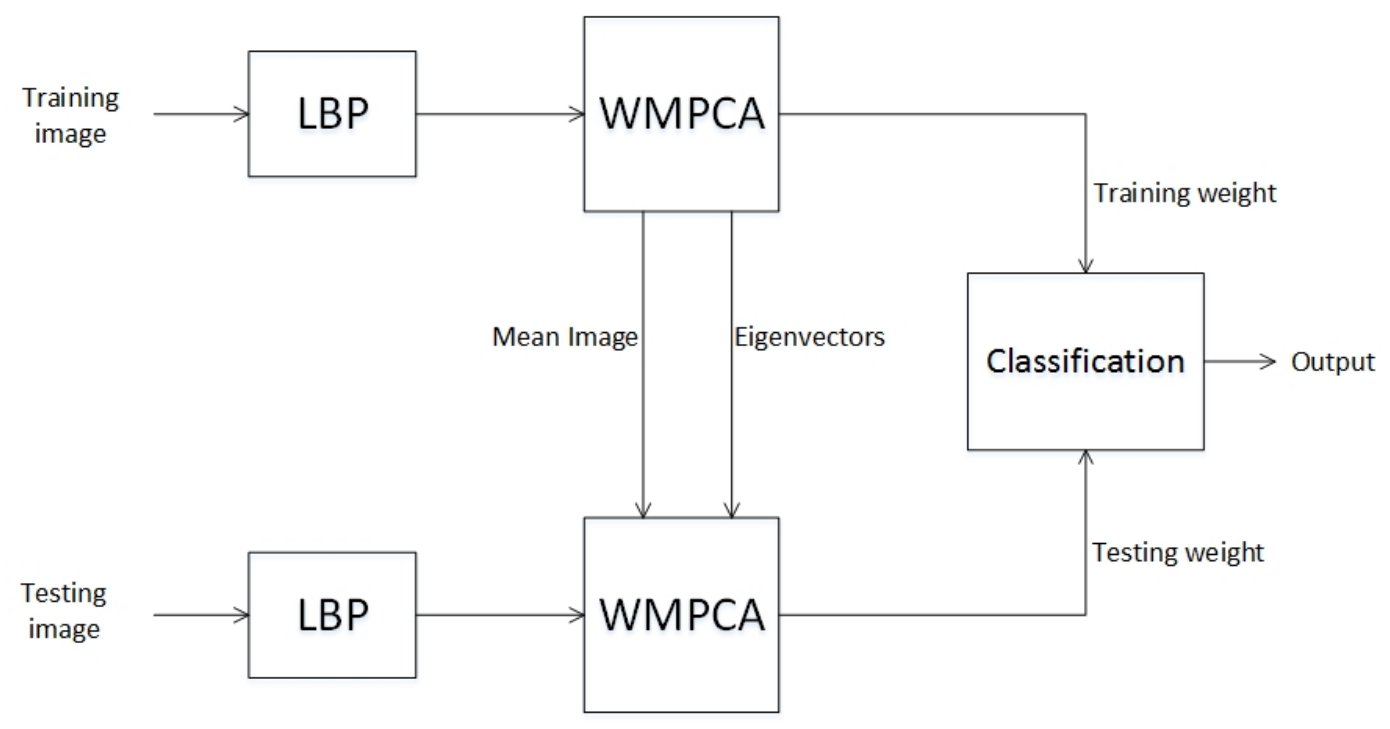

Figure III.1: Algorithm Architecture

\section{III.2 LOCAL BINARY PATTERN (LBP)}

The basic LBP is introduced by Ojala et al. [45], it is presented as a two-level version of the texture unit [46] to describe the local textural patterns. The original structure of LBP works in a 3 by 3 pixel block in an image. The intensity values of the neighbor pixels in the block are thresholded by that of the center pixel. In the 3 by 3 pixel block, there are 8 neighbors around the center pixel, so that a total number of $2^{8}=256$ different labels can be calculated by the relation between the value of the center pixel and that of its neighbors [16]. The basic LBP operator is illustrated in figure III.2. If the starting point is set to the top left corner, the 8-bit code will be 00111001 in counterclockwise direction, which represents 57 in the LBP space. The examples of original image and LBP image are shown in figure III.3, as well as the histogram. 


\begin{tabular}{|c|c|c|}
\hline 6 & 12 & 8 \\
\hline 3 & 10 & 9 \\
\hline 17 & 15 & 10 \\
\hline
\end{tabular}

\begin{tabular}{|l|l|l|}
\hline 0 & 1 & 0 \\
\hline 0 & & 0 \\
\hline 1 & 1 & 1 \\
\hline
\end{tabular}

(a) Original intensity value (b) Thresholded by center pixel

Figure III.2: The basic LBP operator

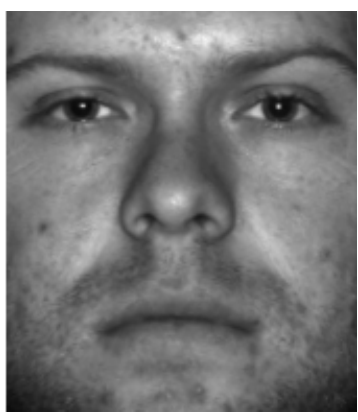

(a) Original image

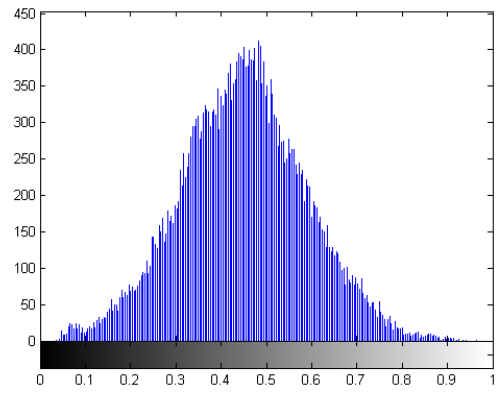

(c) Histogram of original image

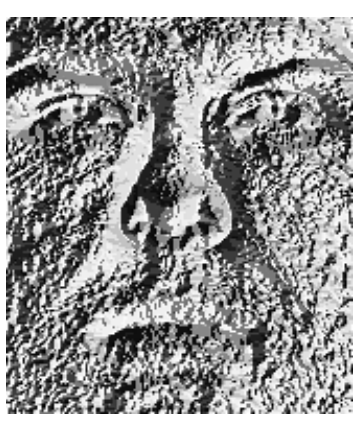

(b) LBP image

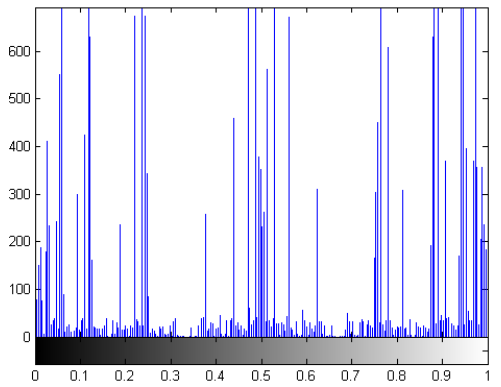

(d) Histogram of LBP image

Figure III.3: Comparison with the original image and its LBP image

After the basic LBP being presented, Ojala et al. made a more generic revised form - there is no limitation of the size of the neighborhood and the number of the sampling points [47], see figure III.4. 

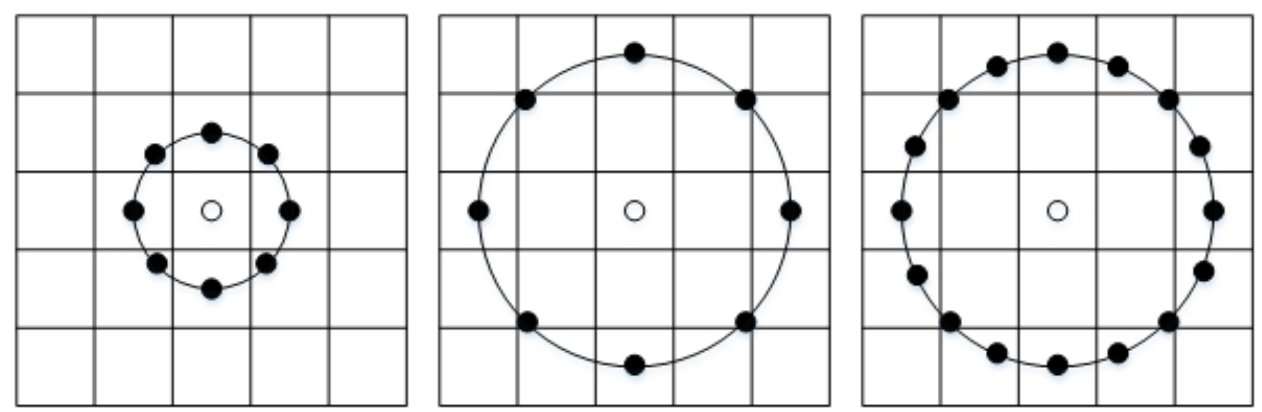

Figure III.4: Sampling points in $(8,1),(8,2),(16,2)$ neighborhood. The points which are not at the block center are interpolated by bilinear interpolation [16]

Consider one pixel $I(x, y)$ in the center part of an image (Due to the size of the pixel block, the pixels on the edge part may "lose" some sampling points), and $g_{c}$ is the intensity value of the pixel. $P$ is represented the number of sampling points, and $R$ is the radius of the neighborhood of $I(x, y)$. The pixel coordinates of the $p$ th sampling point $\left(x_{p}\right.$ and $\left.y_{p}\right)$ are calculated as below:

$$
\begin{aligned}
& x_{p}=x+R \cos (2 \pi p / P), p=0,1,2, \ldots, P-1 \\
& y_{p}=y-R \sin (2 \pi p / P) .
\end{aligned}
$$

The intensity value of the $p$ th sampling pixel $g_{p}$ is:

$$
g_{p}=I\left(x_{p}, y_{p}\right), p=0,1,2, \ldots, P-1
$$

Next, the new value of every pixel needs to be calculated - using the intensity value of every sampling point to subtract the center pixel value, then if the value of the subtraction is equal to or larger than zero, recording the final value to be one, otherwise, zero:

$$
z_{p}=g_{p}-g_{c}
$$




$$
s\left(z_{p}\right)= \begin{cases}1 & \text { if } z_{p} \geq 0 \\ 0 & \text { if } z_{p}<0\end{cases}
$$

where $z_{p}$ is the difference between $g_{p}$ and $g_{c} . \quad s\left(z_{p}\right)$ is the binary value of the $p$ th sampling point after being thresholded. Then, the weighted summation of $s\left(z_{p}\right)$ is calculated. The $L B P_{P, R}$ operator is presented as:

$$
L B P_{P, R}\left(x_{c}, y_{c}\right)=\sum_{p=0}^{P-1} s\left(z_{p}\right) 2^{p}
$$

where $x_{c}$ and $y_{c}$ are the pixel coordinates of the center pixel. Eq. III.6 gives the new label for the center pixel. If $\mathrm{P}$ is equal to 8 , the new range of every pixel is 0 to 255 . One more thing should be considered, the sampling points in the general definition are circularly around the center pixel, whereas the sampling points in the basic LBP are the center part of every small pixel block in the neighborhood. In the general case, the intensity values of the interpolated sampling points need to be calculated.

\section{Bilinear Interpolation}

In this section, a short explanation is made for the bilinear interpolation which is used for calculating the value of the sampling points whose positions are not at the center of their pixel blocks. As illustrate in figure III.5, the value of point $(x, y)$ needs to be calculated by the four neighbor points whose values are known.

In our case, the values of the four neighbor points are the intensity values of the four neighbor pixels around the unknown point. The weighted ratio of every neighbor 


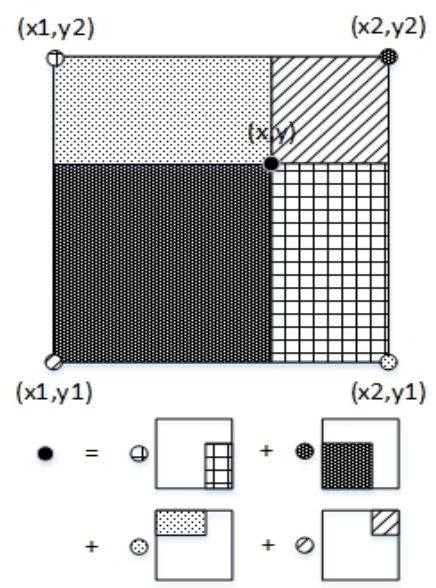

Figure III.5: The geometric representation for bilinear interpolation [48]

point is shown in figure III.5, and the calculation equation can be written as:

$$
\begin{aligned}
f(x, y) \approx \frac{1}{\left(x_{2}-x_{1}\right)\left(y_{2}-y_{1}\right)}( & f\left(x_{1}, y_{2}\right)\left(x_{2}-x\right)\left(y-y_{1}\right) \\
+ & f\left(x_{2}, y_{2}\right)\left(x-x_{1}\right)\left(y-y_{1}\right) \\
& +f\left(x_{2}, y_{1}\right)\left(x-x_{1}\right)\left(y_{2}-y\right) \\
& \left.+f\left(x_{1}, y_{1}\right)\left(x_{2}-x\right)\left(y_{2}-y\right)\right)
\end{aligned}
$$

where $f(x, y), f\left(x_{1}, y_{1}\right), f\left(x_{1}, y_{2}\right), f\left(x_{2}, y_{1}\right), f\left(x_{2}, y_{2}\right)$ are the intensity values of the points $(x, y),\left(x_{1}, y_{1}\right),\left(x_{1}, y_{2}\right),\left(x_{2}, y_{1}\right),\left(x_{2}, y_{2}\right)$ respectively. Using Eq. III.7, the values of the interpolated points can be easily computed.

\section{Uniform Patterns}

Uniform patterns are the extension of the general LBP, and defined by uniform measure of patterns. Uniform measure calculates the number of bitwise transitions from zero to one or one to zero (Figure III.6). A local binary pattern can be called uni- 
form if the uniform measure is at most two [16]. For instance, the patterns 01000000 , uniform measure of 2 , is a uniform pattern, whereas, 01010000 , uniform measure of 4 , is a non-uniform pattern. In uniform LBP operators, every uniform pattern has a unique label, and all the non-uniform patterns are set to one single label. Because of this procedure, the number of labels for uniform patterns can be easily calculated. Consider the number of sampling points is $P$, then the number of labels for uniform patterns is $P(P-1)+3$. It means that there are 59 labels with 8 sampling points, and 243 labels with 16 sampling points. Figure III.7 shows the strategy of obtaining labels for uniform patterns. "ulabel" represents the label for the uniform patterns. The first ulabel is " 0 ".

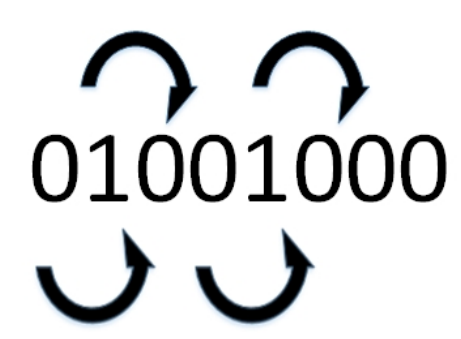

Figure III.6: Calculation strategy for uniform measure: in this sequence, there are 4 bitwise transitions from 1 to 0 or 0 to 1

Uniform patterns are used because that most of the local binary patterns are uniform in natural images. Ojala et al. noticed from the experiments with texture images - around $90 \%$ local binary patterns are uniform in the $(8,1)$ neighborhood, and around $70 \%$ in the $(16,2)$ neighborhood [16]. The experiments with the face images also get the similar results - $90.6 \%$ local binary patterns are uniform in $(8,1)$ neighborhood, and $85.2 \%$ in $(16,2)$ neighborhood. "8" and "16" are represented the number of sampling points, and "1" is the radius. Another reason for using is the statistical robustness of the uniform patterns [16]. Uniform patterns are less prone to 


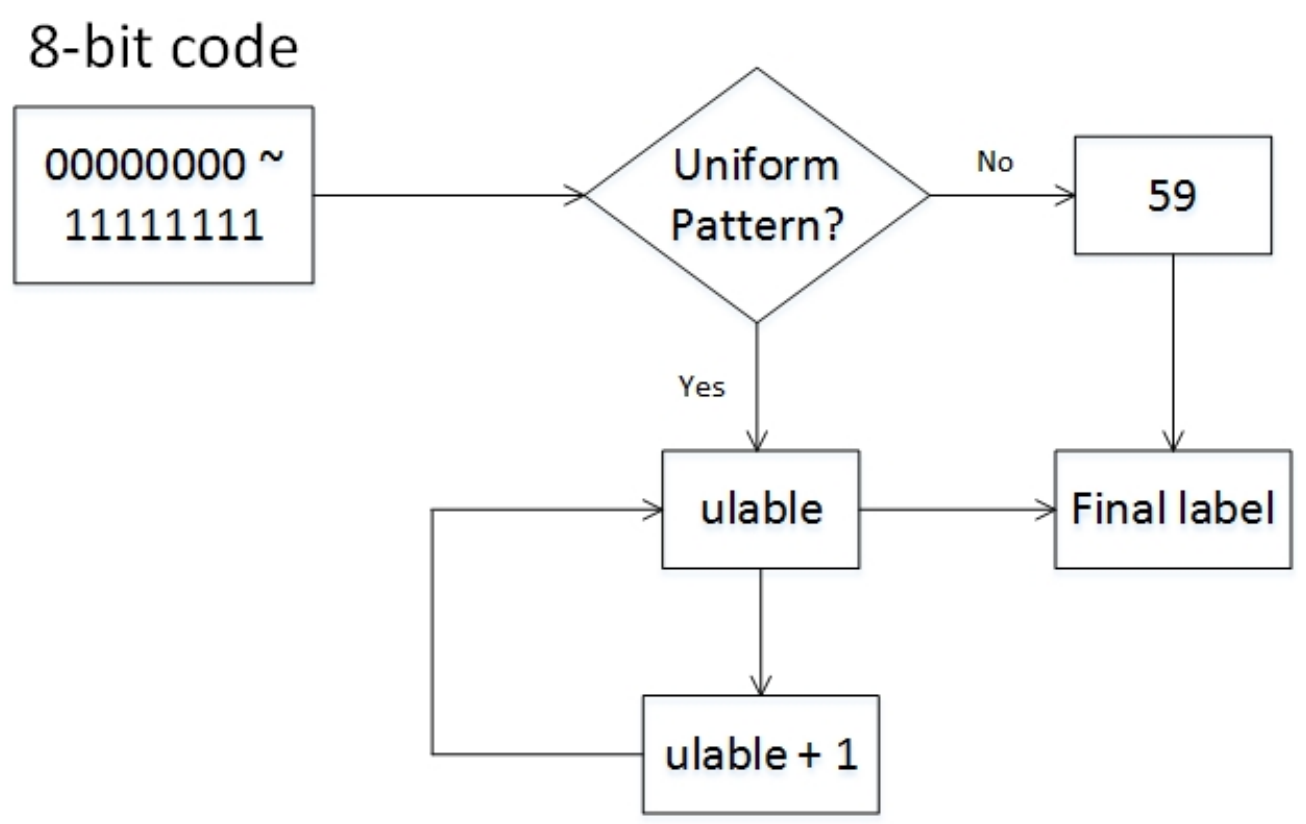

Figure III.7: The strategy for setting the label of uniform and non-uniform patterns (the first value of ulable is 0 )

noise and reduce amount number of labels. It can avoid some redundancies created by the general LBP.

\section{III.3 LOCAL TEXTURAL FEATURE WEIGHTING STRATEGY FOR OCCLUSION INVARIANT REPRESENTATION}

In this section, principal component analysis (PCA) is presented, and a weighting strategy is proposed for dimensionality reduction. PCA is chosen for variable reduction for less processing time, and it also lessens the affection by the redundancy from the original data. In this section, the original algorithm of PCA, modular PCA, and weighted modular PCA are introduces. Weighted modular PCA is the proposed dimensionality reduction method in our facial feature extraction system.

Before the algorithms are presented, the effects of the training and testing system 
should be pointed out. Training system uses a proper algorithm to let the system "learn" from a bunch of training data, then applying the trained rule to the testing system to process the testing data.

\section{PCA}

In principal component analysis, the number of principal components is less than or equal to the variables being considered. As was mentioned before, the first component has the largest possible variance. Eigenvalues represents the amount of variance that is accounted by a given component [49]. Using the meaningful components, which related eigenvalues are larger, helps to get a better result in classification. A larger eigenvalue means a bigger variability of the related principal component. Choosing the components, whose eigenvalues belong to the $K$ largest eigenvalues, will make sense. Eq. III.8 shows the strategy of deciding the value of $K$ :

$$
\text { Percentage }=\frac{\sum_{k=1}^{K} \text { Eigenvalue }_{k}}{\sum_{a=1}^{A} \text { Eigenvalue }_{a}}
$$

where $A$ is the total number of eigenvalues. The proper eigenvalues are chosen when Percentage is larger than a predefined threshold (typically $90 \%$ or more). Then, the processed data (training and testing, will be mentioned below) are combined with the matrix which contains the chosen eigenvectors. The final output values are prepared for classification.

The strategy is presented here. Consider D labeled images of G classes are used for training, the size of every image is $\mathrm{N}$ by M. Before starting the mathematical part, the image matrix needs to be changed to a vector, so that the image size is changed from $\mathrm{N}$ by $\mathrm{M}$ to 1 by NM. The first step is calculating the mean image vector of all 
the training image vectors $\left(\mathbf{I}_{d}\right.$ is the $d$ th training image vector, $\left.d=1,2,3, \ldots, D\right)$ :

$$
\overline{\mathbf{I}}=\frac{1}{D} \sum_{d=1}^{D} \mathbf{I}_{d}
$$

Then, the mean image vector $\overline{\mathbf{I}}$ needs to be subtracted from every training image vector to obtain the distance $\mathbf{I}_{d}^{\text {new }}$ :

$$
\mathbf{I}_{d}^{\text {new }}=\mathbf{I}_{d}-\overline{\mathbf{I}}
$$

Now, compute the covariance matrix $\mathbf{C}$ which is used for extracting eigenvalues and eigenvectors:

$$
\mathbf{C}=\frac{1}{D} \sum_{d=1}^{D} \mathbf{I}_{d}^{\text {new }} \mathbf{I}_{d}^{\text {new } T}
$$

Next step is computing the eigenvalues and eigenvectors of the covariance matrix $\mathbf{C}$ from Eq. III.12:

$$
\mathbf{C v}=\lambda \mathbf{v}
$$

where $\mathbf{v}$ is the eigenvector and $\lambda$ is the eigenvalue. After obtaining all the eigenvectors and eigenvalues, choosing the $K$ largest eigenvalues with Eq. III.8, then using their related eigenvectors to calculate the final training weight outputs $w$ :

$$
w=\left[\sum_{i=1}^{N \times M} v_{K i} I_{1 i}^{n e w}, \sum_{i=1}^{N \times M} v_{K i} I_{2 i}^{n e w}, \ldots, \sum_{i=1}^{N \times M} v_{K i} I_{d i}^{n e w}, \ldots, \sum_{i=1}^{N \times M} v_{K i} I_{D i}^{\text {new }}\right]
$$

where $v_{K i}$ stands for the $i$ th column of $\mathbf{v}_{K}, \mathbf{v}_{K}$ is the matrix combined by the chosen eigenvectors, every row of $\mathbf{v}_{K}$ is an eigenvector. The size of $v_{K i}$ is $K$ by 1 . $I_{d i}^{\text {new }}$ means the $i$ th point in vector $\mathbf{I}_{d}^{\text {new }}$. Now, the testing system can be started with the trained "rule" - chosen eigenvectors $\mathbf{v}_{K}$. Consider there are $D_{\text {test }}$ testing images $\left(\mathbf{I}_{\text {test }}\right)$ with 
the size of $\mathrm{N}$ by $\mathrm{M}$, the size needs to be changed in the same way as that for the training images. Then, the distance $\left(\mathbf{I}_{\text {test }}^{\text {new }}\right.$ test $\left._{\text {th }}\right)$ between the testing image vectors and the mean image vector, obtained in the training system are calculated:

$$
\mathbf{I}_{\text {test }}^{\text {new }} d_{\text {test }}=\mathbf{I}_{\text {test }_{d_{\text {test }}}}-\overline{\mathbf{I}}
$$

where $\mathbf{I}_{\text {test }_{d_{\text {test }}}}$ is the $d_{\text {test }}$ th testing image. The same procedure is used for calculating the final testing weight outputs $w_{\text {test }}$ :

$w_{\text {test }}=\left[\sum_{i=1}^{N \times M} v_{K i} I_{\text {test }_{1} \text { test }_{i}}^{n e w}, \sum_{i=1}^{N \times M} v_{K i} I_{\text {test }_{\text {test }_{i}}}^{\text {new }}, \ldots, \sum_{i=1}^{N \times M} v_{K i} I_{\text {test }_{\text {test }}}^{\text {new }}, \ldots, \sum_{i=1}^{N \times M} v_{K i} I_{\text {test }_{\text {Dtest }}}^{\text {new }}\right]$

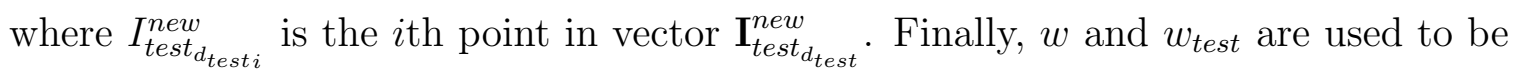
the training and testing data for the classification system.

\section{Modular PCA}

PCA considers the global information of images, but it does not work well when lighting and pose problems exist [18]. Modular PCA separates the original images into several equal modules, then applying PCA for every module, so that the principal components obtained by modular PCA is more representative than PCA. Even though LBP space is used for the feature extraction system, the illumination differences of the lighting variations images are still there. Also, the eigenvectors and eigenvalues of less dimensional covariance matrix are faster to be computed by MATLAB. The procedure for modular PCA is very like PCA, but every step of the algorithm needs to be applied for every module. Consider the training and testing images, which used in PCA, are still being used in modular PCA. First, every image needs to be divided 
into L modules, see figure III.8, and the size of every module is $\mathrm{n}$ by $\mathrm{m}$.
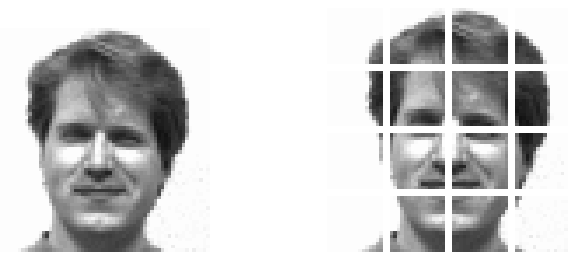

(a) Original image (b) Moduled image

Figure III.8: A face image divided into 16 modules

$\mathbf{I}_{d_{l}}$ is represented the $l$ th module vector of the $d$ th training image vector (size is changed to 1 by nm). Next, the mean module image vector $\overline{\mathbf{I}}_{\text {module }}$ needs to be calculated:

$$
\overline{\mathbf{I}}_{\text {module }}=\frac{1}{D \times L} \sum_{d=1}^{D} \sum_{l=1}^{L} \mathbf{I}_{d_{l}}
$$

Then, $\overline{\mathbf{I}}_{\text {module }}$ needs to be subtracted from $\mathbf{I}_{d_{l}}$ :

$$
\mathbf{I}_{d_{l}}^{\text {new }}=\mathbf{I}_{d_{l}}-\overline{\mathbf{I}}_{\text {module }}
$$

where $\mathbf{I}_{d_{l}}^{\text {new }}$ is the difference between $\mathbf{I}_{d_{l}}$ and $\overline{\mathbf{I}}_{\text {module. Now, the covariance matrix }}$ $\mathbf{C}_{\text {module }}$ of all the modules from all the images needs to be calculated:

$$
\mathbf{C}_{\text {module }}=\frac{1}{D \times L} \sum_{d=1}^{D} \sum_{l=1}^{L} \mathbf{I}_{d_{l}}^{\text {new }} \mathbf{I}_{d_{l}}^{\text {new } T}
$$

With the covariance matrix, all the eigenvectors and eigenvalues are computed by Eq. III.19:

$$
\mathbf{C}_{\text {module }} \mathbf{v}_{\text {module }}=\lambda_{\text {module }} \mathbf{v}_{\text {module }}
$$

where $\mathbf{v}_{\text {module }}$ represents the eigenvector, $\lambda_{\text {module }}$ represents the eigenvalue. With the 
same procedure in PCA, the final training weight outputs $(w)$ can be calculated:

$$
\begin{array}{r}
w=\left[\sum_{i=1}^{n \times m} v_{\text {module } K i} I_{1_{1 i}}^{n e w}, \sum_{i=1}^{n \times m} v_{\text {module } K i} I_{1_{2 i}}^{n e w}, \sum_{i=1}^{n \times m} v_{\text {module } K i} I_{1_{3 i}}^{n e w}, \ldots, \sum_{i=1}^{n \times m} v_{\text {module } K i} I_{1_{L i}}^{\text {new }},\right. \\
\left.\ldots, \sum_{i=1}^{n \times m} v_{\text {module } K i} I_{d_{l i}}^{n e w}, \ldots, \sum_{i=1}^{n \times m} v_{\text {module } K i} I_{D_{1 i}}^{\text {new }}, \ldots, \sum_{i=1}^{n \times m} v_{\text {module } K i} I_{D_{L i}}^{\text {new }}\right]
\end{array}
$$

where $v_{\text {module } K i}$ is the $i$ th column of $\mathbf{v}_{\text {module } K}, \mathbf{v}_{\text {module } K}$ is the matrix combined by the chosen eigenvectors, every row of $\mathbf{v}_{\text {module } K}$ is an eigenvector, and the size of $v_{\text {module } K i}$ is $K$ by $1 . I_{d_{l i}}^{\text {new }}$ is the $i$ th point in vector $\mathbf{I}_{d_{l}}^{\text {new }}$. For testing system, every testing image is also divided into L modules. Following the same strategy as the one in PCA, the final testing weight outputs $\left(w_{\text {test }}\right)$ are here:

$$
\begin{aligned}
& w_{\text {test }}=\left[\sum_{i=1}^{n \times m} v_{\text {module Ki }} I_{\text {text }_{1 \text { test } 1 i}}^{\text {new }}, \sum_{i=1}^{n \times m} v_{\text {module Ki }} I_{\text {text }_{1 \text { test } 2 i}}^{\text {new }}, \sum_{i=1}^{n \times m} v_{\text {module Ki }} I_{\text {text }_{1}}^{\text {new } \text { test } 3 i},\right. \\
& \ldots, \sum_{i=1}^{n \times m} v_{\text {module Ki }} I_{\text {text }_{1} \text { test } L i_{i}}^{n e w}, \ldots, \sum_{i=1}^{n \times m} v_{\text {module Ki }} I_{\text {text }_{d_{\text {test } i}}^{\text {new }}} \text {, } \\
& \left.\ldots, \sum_{i=1}^{n \times m} v_{\text {module Ki }} I_{\text {text }_{D_{\text {test } 1 i}}^{\text {new }}}, \ldots, \sum_{i=1}^{n \times m} v_{\text {module Ki }} I_{\text {text }_{D_{\text {test } L i}}^{\text {new }}}\right]
\end{aligned}
$$

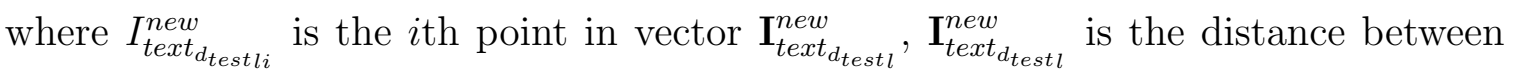
the $l$ th module of the $d$ th testing image vector and the mean module image vector obtained from the training part. The outputs of the modules, which belong to the same image, need to be considered as one output, so that the whole weight output 
matrix in training $(w)$ and testing $\left(w_{\text {test }}\right)$ parts are shown respectively as below:

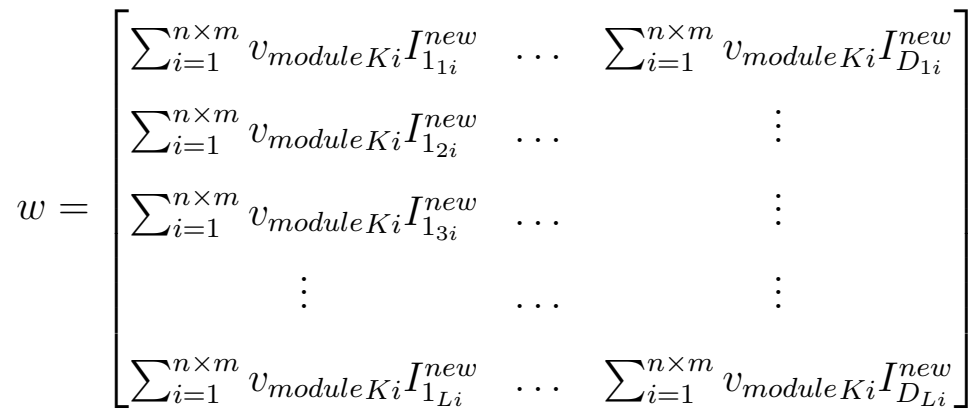

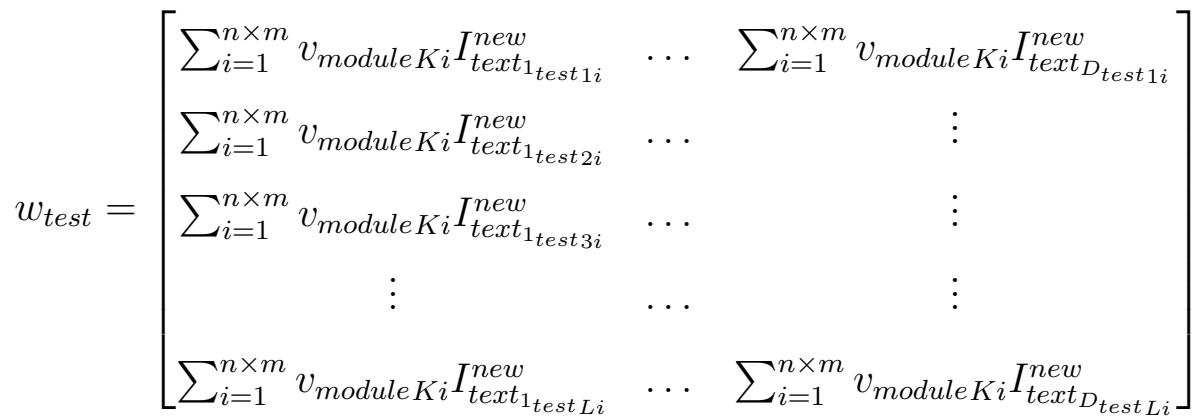

Every column represents the information extracted from one image. Figure III.9 shows the reconstructed images after applying PCA and MPCA with the same number of eigenvectors, the reconstruction of MPCA is clearer. From the recognition result in [18], a clear comparison between PCA and MPCA is obtained. In the experiment, 8 images of every individual (Yale database) are used for training and the other 3 images are used for testing. MPCA shows a better recognition result than PCA when using the same number of eigenvectors. Also, the best recognition result of MPCA (nearly $90 \%)$ is higher than that of PCA (70\%). 


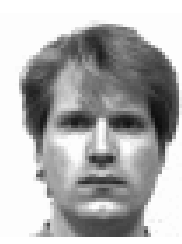

(a) Original testing image

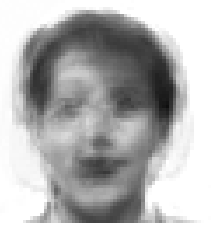

(b) Reconstructed image from the weight outputs obtained by PCA

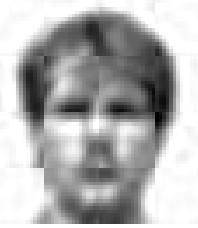

(c) Reconstructed image from the weight outputs obtained by MPCA (16 modules)

Figure III.9: The comparison between the reconstructed images from PCA and MPCA

\section{Weighted Modular PCA}

Weighted modular PCA is more effective when some parts of an image are occluded or in bad lighting conditions (just part of the image, not the whole image). Every module is weighted by the variance of its related region to minimize the affection of the parts with occlusions or bad lighting conditions, so that there are L weights for every image, and every element in the same module is weighted by a same weight. $\operatorname{var}_{d_{\text {test }} l}=E\left[\left(\mathbf{I}_{\text {test }_{d_{\text {test }}}}-\overline{\mathbf{I}}_{\text {test }_{\text {module }}}\right)^{2}\right]\left(E[]\right.$ means the mean value, $\mathbf{I}_{\text {test }_{d_{\text {test }}}}$ means the $l$ th module vector of the $d_{\text {test }}$ th testing image, $\overline{\mathbf{I}}_{\text {test }}$ module means the mean testing image module vector) defines the variance/weighting strategy for the $l$ th module of the 
$d_{\text {test }}$ th testing image. The final weight outputs are changed as below:

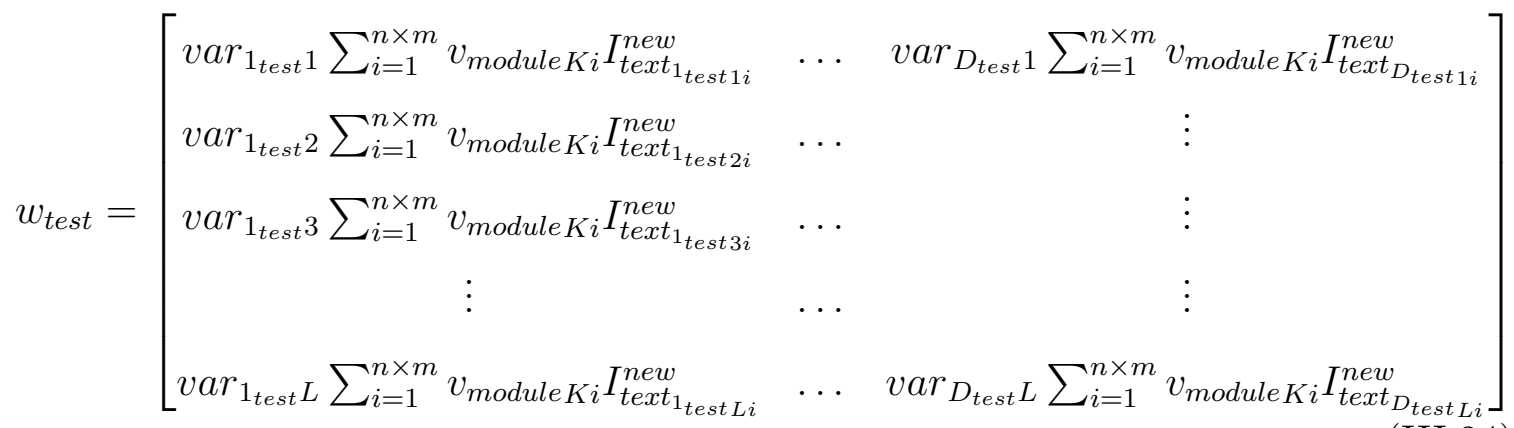

One more thing needs to be mentioned: calculate the variance of every module for the testing images rather than the training images. The training procedure is the same as modular PCA, and the weighting strategy used for classification are more described in section III.4.

\section{III.4 CLASSIFICATION}

After facial feature extraction by LBP and dimensionality reduction by WMPCA, the weight outputs ( $w$ and $w_{\text {test }}$ from WMPCA) are used for classification. Depending on the feature property and the system efficiency, Euclidean distance is used for classification. First the average weight output of the training images in the same class needs to be calculated:

$$
\bar{w}_{g}=\frac{1}{F} \sum_{f=1}^{F} w_{g f}
$$

where $\bar{w}_{g}$ is the average value of $g$ th class of the weight outputs in training patterns $(w) . F$ is the total number of training patterns in $g$ th class. $f=1,2,3, \ldots, F$. Then, the distances between the average value in every class and the testing weight outputs 
are calculated:

$$
\operatorname{Dist}_{d_{\text {test } g}}=\frac{1}{N M} \sqrt{\sum_{i=1}^{N M}\left(\operatorname{Var}_{d_{\text {test } i}} \bar{w}_{g_{i}}-w_{\text {test }_{\text {test } i}}\right)^{2}}
$$

$$
\begin{array}{r}
\operatorname{Var}_{d_{\text {test }}}=[\underbrace{\operatorname{var}_{d_{\text {test }} 1}, \operatorname{var}_{d_{\text {test }} 1}, \ldots, \operatorname{var}_{d_{\text {test }} 1}}_{N M / L}, \underbrace{\operatorname{var}_{d_{\text {test }} 2}, \operatorname{var}_{d_{\text {test }} 2}, \ldots, \operatorname{var}_{d_{\text {test } 2}}}_{N M / L}, \\
\ldots, \ldots, \underbrace{\operatorname{var}_{d_{\text {test }} L}, \operatorname{var}_{d_{\text {test }} L}, \ldots, \operatorname{var}_{d_{\text {test }} L}}_{N M / L}]^{T}
\end{array}
$$

$\mathbf{I}_{\text {text }_{d_{\text {test }}}}$ belong to the class which Dist $_{d_{\text {test }}}$ is the minimum, and Dist $d_{d_{\text {test }}}<c . c$ is a small threshold value chosen by experiments. $\operatorname{Var}_{d_{\text {test }}}$ is the vector of all the variances for the $d_{\text {test }}$ th testing image vector. $V a r_{d_{t e s t} i}$ is the $i$ th variance in $\operatorname{Var}_{d_{t e s t}}$.

\section{III.5 SUMMARY}

In this chapter, the proposed face recognition technique is presented. First, the local binary pattern is introduced. The improvement due to the basic LBP is shown. Second, the principal component analysis is discussed. The proposed method uses a more effective version - weighted modular PCA for dimensionality reduction. The basic algorithms are presented in detail. In the next chapter, an enhanced version of LBP is introduced to the proposed system, making the system more robust. 


\section{CHAPTER IV}

\section{ENHANCED LOCAL TEXTURAL REPRESENTATION FOR FACE RECOGNITION}

In Chapter III, the proposed method improves the performance, especially under different lighting conditions. For enhancing the ability of general LBP, we proposed an enhanced version based on the general LBP. The enhanced method makes the system more effective. Figure IV.1 shows the frame work of the enhanced proposed method.

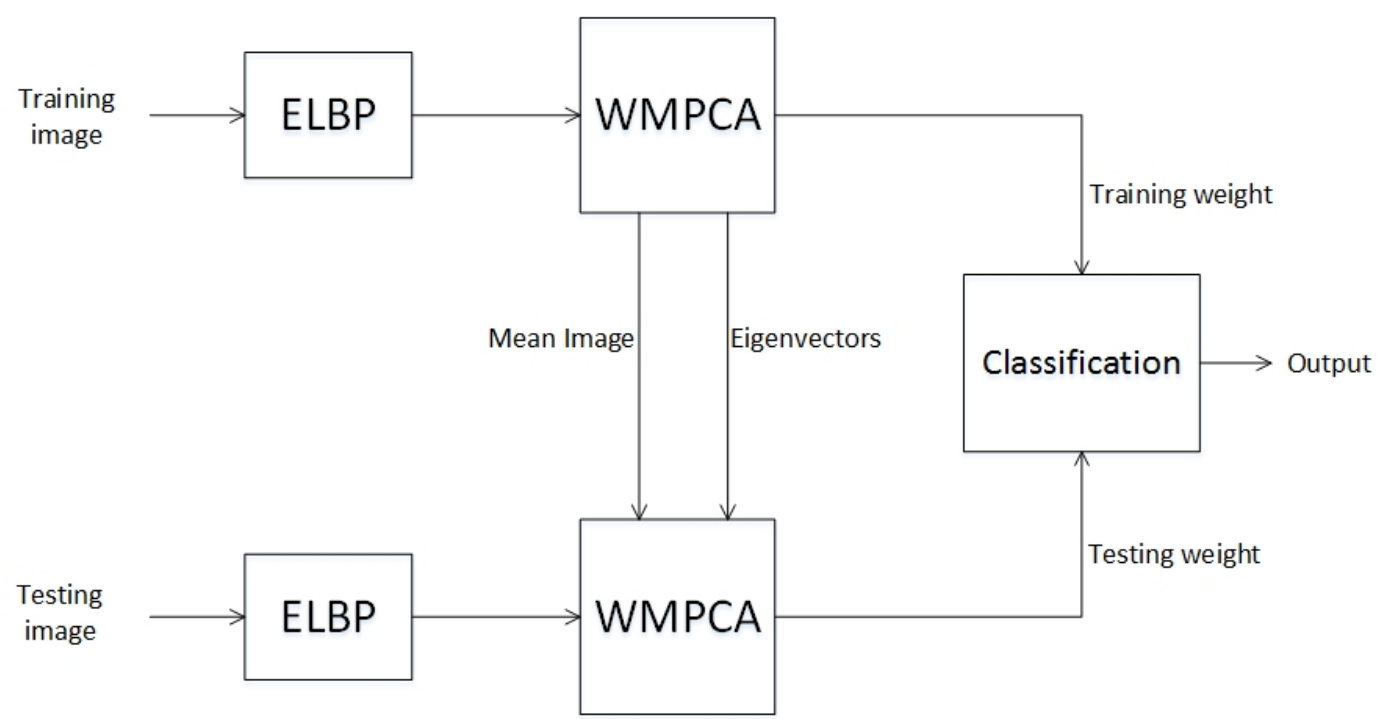

Figure IV.1: The frame work of the enhanced proposed method 


\section{IV.1 ENHANCED LBP (ELBP)}

ELBP can extract texture information in extreme dark or bright conditions caused by lighting issue. General LBP calculates the new label of every pixel by using the relationship with its related neighbors, so that it may be coded "0" as "1" or vice versa when there are small variations in the neighborhood. This may cause more errors when compare with the LBP image in the neutral lighting conditions. Instead of thresholding every neighbor pixel by comparing its intensity value with that of the center pixel directly, the procedure uses the comparison of the summation of the positive distance and the total absolute distance between the intensity value of the neighbor pixels and that of the center pixel to threshold (absolute distance of a sampling point: the distance between the sampling point and its related center pixel without sign; positive distance of a sampling point: if the sign of the distance between the sampling point and its related center point is positive, the positive distance is equal to the absolute distance, other wise, 0). For considering the affection of all the neighbor pixels, first one sampling point (in the neighborhood) is taken into consideration, if the intensity value of the point is bigger than that of the center pixel, the total positive distance $\left(T P D_{1}\right)$ is equal to the total absolute distance $\left(T D_{1}\right)$. Because of $T P D_{1}>\frac{1}{2} T D_{1}$, recording $s_{1}=1$, otherwise, $s_{1}=0$. Then, using another sampling point: this new point should also be in the neighborhood of the center pixel, and it is also the neighbor of the first sampling point in the clockwise direction. Adding the absolute distances of the two sampling points, then adding the positive distances together, and comparing the total absolute distance $\left(T D_{2}\right.$ Eq. IV.1) of the two sampling points with the total positive distance (TPD $D_{2}$ Eq. IV.2). 
If $T P D_{2}>\frac{1}{2} T D_{2}$, recording $s_{2}=1$, otherwise, $s_{2}=0$.

$$
\begin{aligned}
& T D_{q}=\sum_{i=1}^{q}\left|g_{i}-g_{c}\right| \\
& T P D_{q}=\sum_{g_{k} \geq g_{c}}\left(g_{k}-g_{c}\right)
\end{aligned}
$$

where $q=1,2,3, \ldots, P, q$ means the number of sampling points taken into consideration ( $q$ and $p$ have different meanings, but the maximum values are both $P$ ). $k$ is less than or equal to $q$. Next, one more sampling point is taken into consideration, following the same procedure shown as above until all the sampling points are considered. Now, a P-bit code of the center pixel is obtained. Different from the general LBP, this P-bit code has no directions, because only the local relationships between the center pixel and different neighbor areas are considered. Then, the new label of the center pixel is obtained by counting the number of " 1 " $\mathrm{s}$ in the P-bit code. The label is equal to the number of " 1 "s. Consider a 3 by 3 neighborhood is used, see figure IV.2, so that 9 new labels (0 to 8) can be obtained from the 8-bit code. The first sampling point can be chosen from any point in the neighborhood. Figure IV.2 shows the intensity values $\left(g_{1}\right.$ to $\left.g_{8}\right)$ of every pixel $\left(P_{1}\right.$ to $P_{8}$ respectively) in a 3 by 3 neighborhood.

The recording number $s_{q}$ of different numbers of the considered sampling points is thresholded by the relationship between $T D_{q}$ and $T P D_{q}$ shown as below:

$$
s_{q}= \begin{cases}1 & \text { if } T P D_{q}>\frac{1}{2} T D_{q} \\ 0 & \text { if } T P D_{q} \leq \frac{1}{2} T D_{q}\end{cases}
$$




\begin{tabular}{|l|l|l|}
\hline g1 & g2 & g3 \\
\hline g8 & gc & g4 \\
\hline g7 & g6 & g5 \\
\hline
\end{tabular}

Figure IV.2: A 3 by 3 neighborhood: $g_{1}$ to $g_{c}$ are intensity values [50]

If $T P D_{q}>\frac{1}{2} T D_{q}, s_{q}=1$ otherwise, $s_{q}=0$. Finally, the P-bit code can be obtained by $s_{1} s_{2} s_{3} \ldots s_{q} \ldots s_{P}$

\section{IV.2 THE ALGORITHM STEPS FOR THE ENHANCED LBP}

Figure IV.3 shows the frame work of the proposed enhanced LBP.

Step 1: Compute the distance Dist ${ }_{p}$ between the center pixel and its related sampling points, where $p=1,2,3, \ldots, P$ and $P$ is the total number of sampling points. Indicating whether the distance is positive or negative.

Step 2: Obtaining the absolute distances $A D_{p}$ from $\left|D_{i s t}\right|$.

Step 3: Obtaining the positive distances $P D_{p}$ from Dist $_{p}$ (if Dist $_{p}>0, P D_{p}=A D_{p}$, otherwise, $\left.P D_{p}=0\right)$.

Step 4: Calculating the total absolute distance $T D_{1}$ and total positive distance $T P D_{1}$ of the first neighbor pixel $P_{1}$ (consider the starting point is on the top left corner). Step 5: Calculating $T D_{2}$ and $T P D_{2}$ of $P_{1}$ and $P_{2}$. Then, calculating $T D_{3}$ and $T P D_{3}$ of $P_{1}, P_{2}$, and $P_{3}$. Repeating this procedure until all the neighbor pixels are considered $\left(P_{1}, P_{2}, P_{3}, \ldots\right.$ are represented the related sampling points in clockwise direction). 


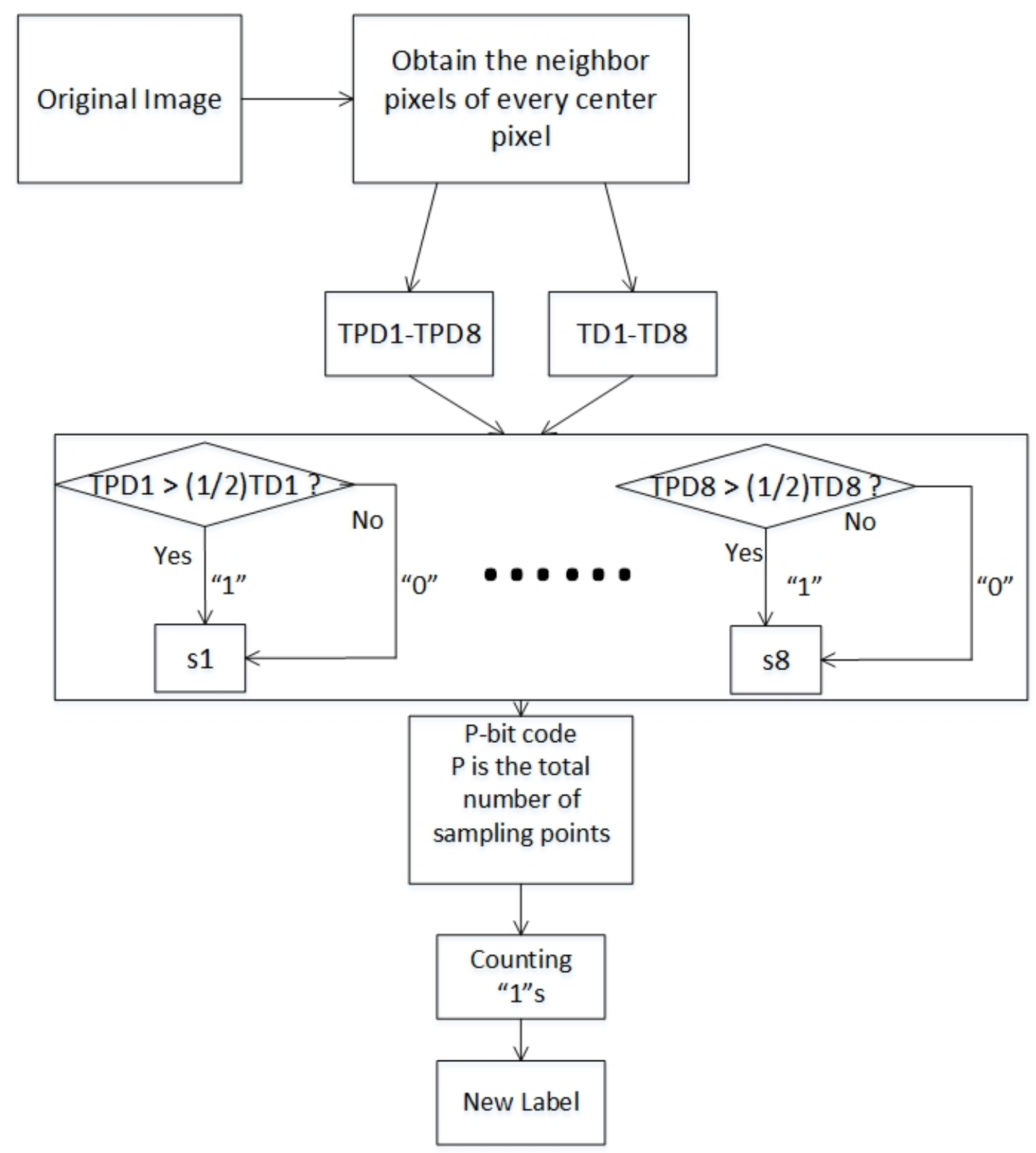

Figure IV.3: The frame work of the proposed enhanced LBP

Step 6: Compared $\frac{1}{2} T D_{q}$ with $T P D_{q}$. If $T P D_{q}>\frac{1}{2} T D_{q}, s_{q}=1$, otherwise, $s_{q}=0$.

Step 7: Obtaining the P-bit binary code from $\left[s_{1} s_{2} s_{3} \ldots s_{P}\right]$.

Step 8: Counting the number of "1"s in the P-bit code, and obtaining the new label from the counting. For example, if there are X "1"s, the new label for the center pixel 
is $\mathrm{X}$, so that there are $\mathrm{P}+1$ new labels, and the first new label is 0 .

Step 9: Now, the original image is represented by the new labels.

Figure IV.4 shows an example with the whole procedure of ELBP.

Eq. IV.4 shows the calculation steps of figure IV.4 (starting at the top left corner):

\begin{tabular}{|c|c|c|}
\hline 17 & 5 & 1 \\
\hline 40 & 9 & 4 \\
\hline 30 & 12 & 15 \\
\hline
\end{tabular}

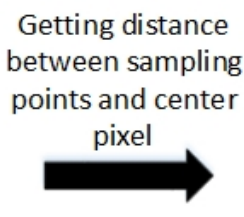

\begin{tabular}{|c|c|c|}
\hline 8 & -4 & -8 \\
\hline 31 & 9 & -5 \\
\hline 21 & 3 & 6 \\
\hline
\end{tabular}

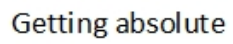
distance between sampling points and center pixel

\begin{tabular}{|c|c|c|c|}
\cline { 2 - 4 } center pixel & 31 & 9 & 5 \\
\cline { 2 - 4 } & 21 & 3 & 6 \\
\hline
\end{tabular}

Getting positive
distance between
sampling points and
center pixel, setting
the negative distance
to be 0
\begin{tabular}{|c|c|c|}
8 & 0 & 0 \\
\hline 31 & 9 & 0 \\
\hline 21 & 3 & 6 \\
\hline
\end{tabular}

Figure IV.4: Example for ELBP 


$$
\begin{aligned}
& T P D_{1}=8, T D_{1}=8, T P D_{1}>\frac{1}{2} T D_{1} \longrightarrow s_{1}=1 \\
& T P D_{2}=8+0=8, T D_{2}=8+4=12, T P D_{2}>\frac{1}{2} T D_{2} \longrightarrow s_{2}=1 \\
& T P D_{3}=8+0+0=8, T D_{3}=8+4+8=20, T P D_{3}<\frac{1}{2} T D_{3} \longrightarrow s_{3}=0 \\
& T P D_{4}=8+0+0+0=8, T D_{4}=8+4+8+5=25, T P D_{4}<\frac{1}{2} T D_{4} \longrightarrow s_{4}=0 \\
& T P D_{5}=8+0+0+0+6=14, T D_{5}=8+4+8+5+6=31, \\
& T P D_{5}<\frac{1}{2} T D_{5} \longrightarrow s_{5}=0 \\
& T P D_{6}=8+0+0+0+6+3=17, T D_{6}=8+4+8+5+6+3=34, \\
& T P D_{6}=\frac{1}{2} T D_{6} \longrightarrow s_{6}=0 \\
& T P D_{7}=8+0+0+0+6+3+21=38, T D_{7}=8+4+8+5+6+3+21=55 \text {, } \\
& T P D_{7}>\frac{1}{2} T D_{7} \longrightarrow s_{7}=1 \\
& T P D_{8}=8+0+0+0+6+3+21+31=69 \text {, } \\
& T D_{8}=8+4+8+5+6+3+21+31=86, T P D_{8}>\frac{1}{2} T D_{8} \longrightarrow s_{8}=1
\end{aligned}
$$

So that the 8-bit code is obtained from Eq. IV.4, $s_{1} s_{2} s_{3} s_{4} s_{5} s_{6} s_{7} s_{8} \longrightarrow 11000011$. Four "1"s appear in the sequence, so the new label of the center pixel is 4 . The 8-bit code is affected by the starting point in the neighborhood, and the best starting points need to be set depending on different databases. Figure IV.5 shows a comparison of an example from Yale B database [3]. More details can be obtained from the ELBP image, such as the edge of eyebrows and eye sockets. There is less comparison than 
the LBP image between left half of face and right half of face caused by lighting angles.

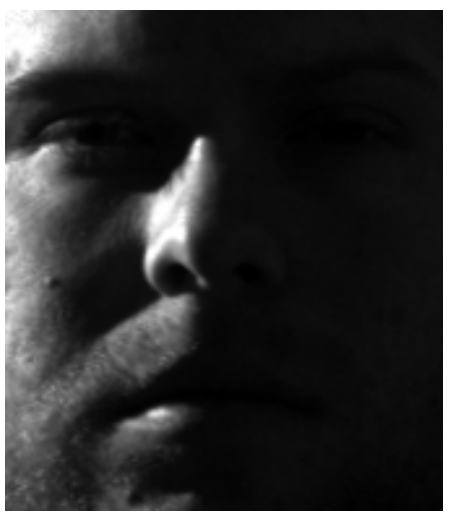

(a) Original image

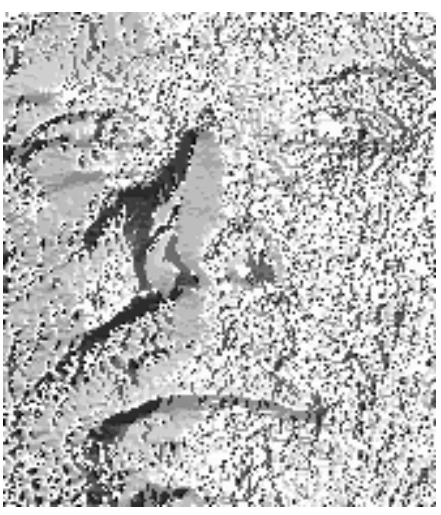

(b) LBP image

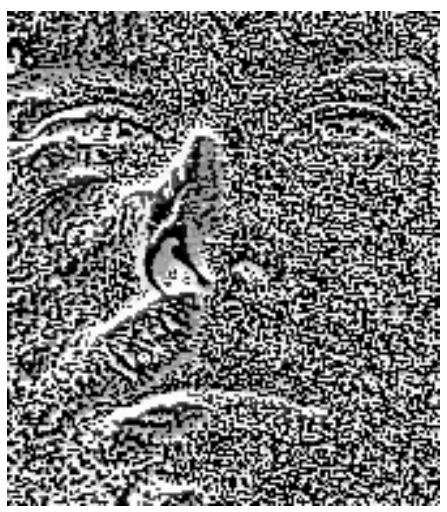

(c) ELBP image

Figure IV.5: The comparison between LBP and ELBP images in a difficult lighting condition

The modified system uses the enhanced local binary patterns and weighted modular PCA for facial feature extraction. Then, applying Euclidean distance for classification. The algorithms for weighted modular PCA and Euclidean distance are the same as the ones was mentioned before.

\section{IV.3 SUMMARY}

In this chapter, the enhanced LBP is presented. The algorithm steps are explained in detail, and the comparison of the output images shown in figure IV.5 illustrates the advantage after applying ELBP to the original image. As one can see, more detailed information can be obtained in poor lighting conditions. In the next chapter, the experimental results show the effectiveness of the proposed method. 


\section{CHAPTER V \\ EXPERIMENTAL RESULT ANALYSIS}

In this chapter, the performance of the proposed method is compared with other algorithms on Yale database, CMU AMP Facial Expression database, Japanese Female Facial Expression database(JAFFE), and Extended Yale B database. This chapter is separated into three parts for experimental result analysis: section V.1 shows the results of face expression variation on multiple databases; section V.2 illustrates the results with difficult lighting conditions; section V.3 analyzes the results with various types of occlusions.

\section{V.1 EXPERIMENTS ON FACIAL EXPRESSION VARIATION DATABASES}

This section shows the comparison between the proposed method and other existing methods on Yale Database, CMU AMP Facial Expression database, and JAFFE database.

\section{Experiments on Yale Database}

The Yale database contains 15 individuals with 11 images of each. All the images are cropped to 64 by 64 . Figure V.1 shows the comparison results between the proposed method and the methods presented in [51]. ELBP3 uses 3 by 3 neighborhood (8 sampling points) to calculate the ELBP image. ELBP5 uses 5 by 5 neighborhood 
(16 sampling points) to calculate the ELBP image. The $\mathrm{x}$ axis represents the number of eigenvectors; the y axis represents the recognition rate. 10, 20, 30, 50, 100, and 150 eigenvectors are used respectively to obtain the result.

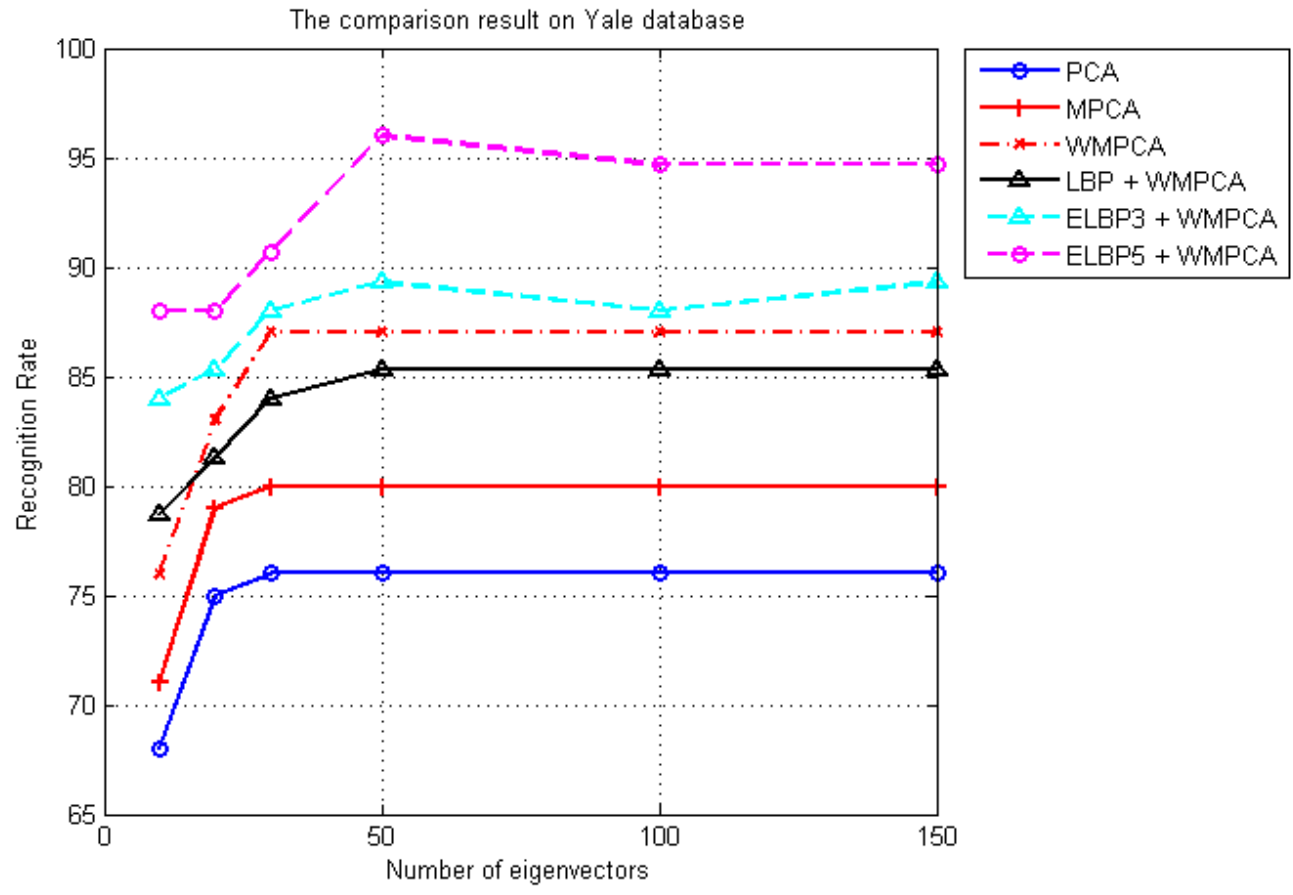

Figure V.1: Performance comparison with Yale database

From figure V.1, the best result is obtained by ELBP5 + WMPCA, which using 50 eigenvectors for every module, and the accuracy is as high as $96 \%$. Also, the best starting point for our adding procedure, shown in figure V.2. Figure V.3 shows the comparison results between the proposed method and partial methods in [52], using the same strategy of doing experiments in [52]. Three images of every individual are randomly chosen to be the training images and all the others to be the testing images. The result of the proposed method is the average result for ten runs. The recognition result of the proposed method is $91.17 \%$. 


\begin{tabular}{|c|c|c|c|c|}
\hline$P 1$ & $P$ & $P 3$ & $P 4$ & $P 5$ \\
\hline$P 16$ & & & & $P 6$ \\
\hline$P 15$ & & & & $P 7$ \\
\hline$P 14$ & & & & $P 8$ \\
\hline$P 13$ & $P 12$ & $P 11$ & $P 10$ & $P 9$ \\
\hline
\end{tabular}

Figure V.2: The order for constructing the 16-bit code: starting point is $P_{3}$ (Yale database)

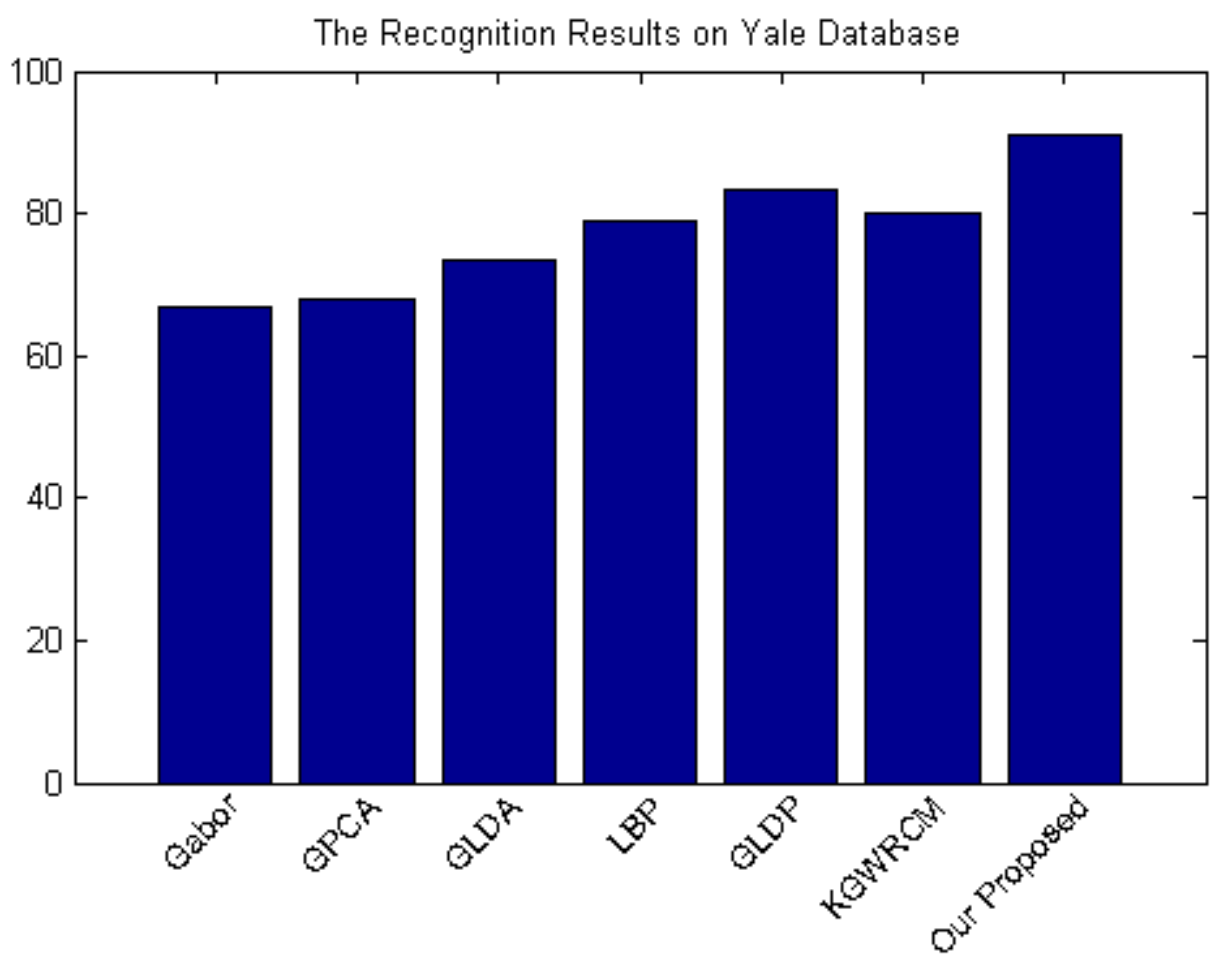

Figure V.3: The comparison with the results in [52] on Yale database

The figure above shows the results with some methods in [53] (GPCA - Gabor PCA and GLDA - Gabor LDA) and [54] (KGWRCM - Kernel Gabor Weighted Region Covariance Matrix). 


\section{Experiments on CMU AMP Facial Expression Database}

CMU AMP Facial Expression database has 13 people and 75 images for each. The size of every image is cropped to 64 by 64 . We randomly choose 10 images per individual to be the training images and the others to be the testing images. The experimental results show that the performance of ELBP3 is better than that of ELBP5 because every image in CMU AMP database cropped just the face part of the entire head, and there is no information about the hair. The comparison with the background is less. If more sampling points were used in a large neighborhood, the face structures would be more clear but the the differences between various individuals might be small, so that ELBP3 is chosen for this database. Figure V.4 shows the starting point for calculating the 8-bit binary patterns. Table V.1 shows the comparison of the recognition results between the proposed method and that in $[55,56,57]$.

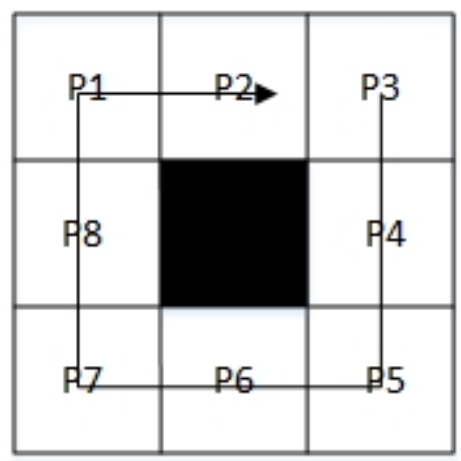

Figure V.4: The order for constructing the 8-bit code: starting point is $P_{3}(\mathrm{CMU}$ AMP Facial Expression database)

Even though the proposed method does not reach the highest accuracy, the advantage is that the computation time is less (2.9044s for the proposed method). The computation costs of other methods do not be mentioned in their papers, but 


\begin{tabular}{|c|c|}
\hline Methods & Recognition Rate \\
\hline Sparse Representation-based Classification [55] & 99.49 \\
\hline Compressive Sensing based [56] & 100 \\
\hline Self organization learning embedded lines of attraction [57] & 100 \\
\hline Our Proposed Method & 99.93 \\
\hline
\end{tabular}

Table V.1: The comparison with the results in $[55,56,57]$ on CMU AMP Facial Expression database

one of them needs to consider choosing the proper parameter in order to balance the accuracy and computation cost. We lose some accuracy but the efficiency is higher.

\section{Experiments on Japanese Female Facial Expression Database}

This database has 213 images of 10 Japanese female individuals. All the images are cropped to 64 by 64 . Every individual has one normal expression and several different expression images. In our experiment, 6 images of every individual are randomly chosen to be the training images and all the others to be the testing images. The starting point for calculating 16-bit code is the same as the one in Yale database. The proposed result (average result for ten runs) shows in figure V.5 compared with the results in [58]. 


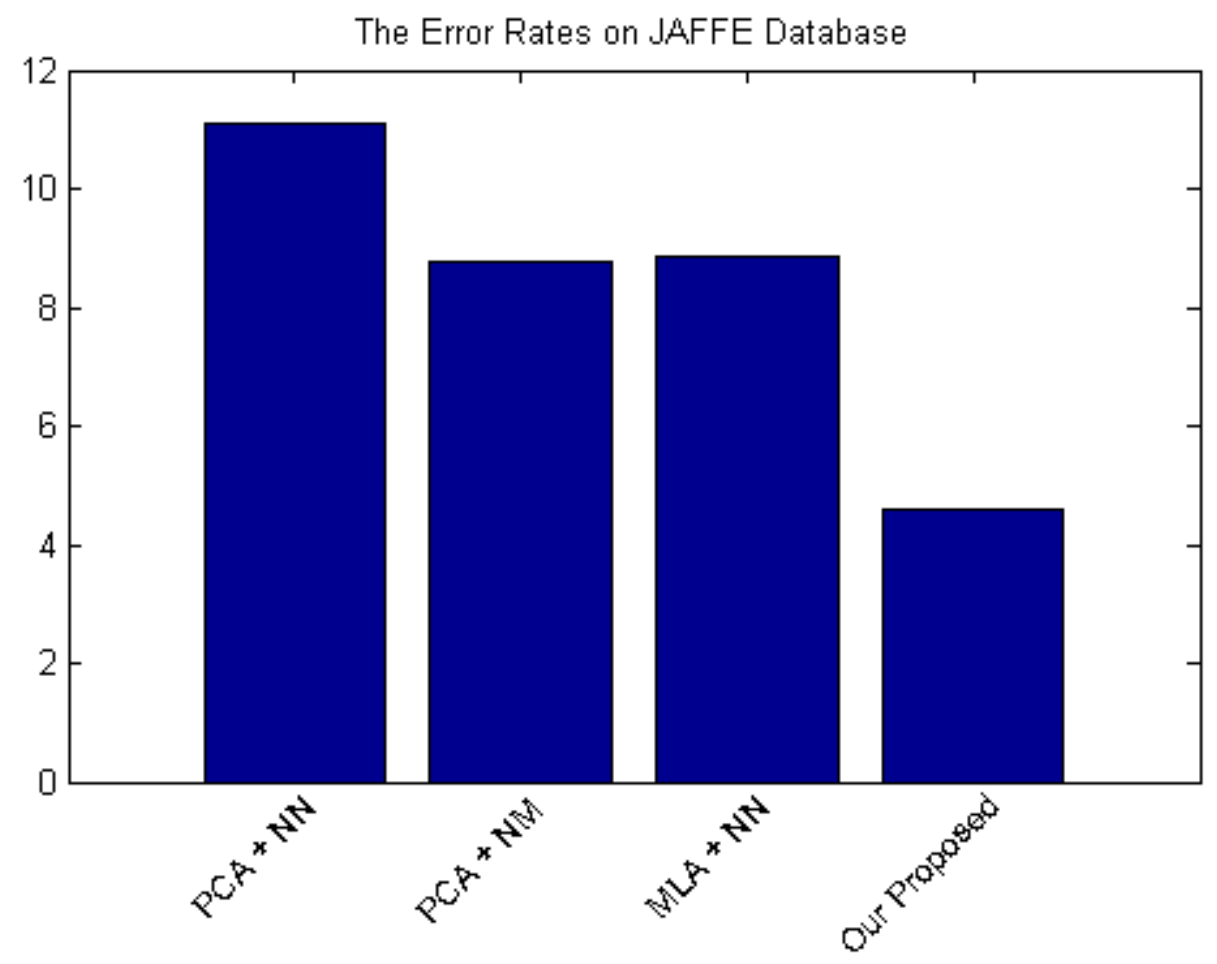

Figure V.5: The error rates on JAFFE database

\section{V.2 EXPERIMENTS ON LIGHTING VARIATION DATABASE (EX- TENDED YALE B DATABASE)}

Lighting issues are hard to resolve for face recognition systems, however, with the proposed algorithm, a better facial feature extraction can be obtained. Extended Yale B database has 38 individuals, and every individual has 64 images with different lighting conditions. All the images are cropped to 64 by 64 . Figure V.6 shows some comparisons between general LBP and ELBP, the images are obtained from Yale B database with different lighting conditions. Table V.2 and table V.3 show the comparison of the recognition rates with [59]. Seven images of every individual are used for training, and all the others are used for testing. This experiment is separated 


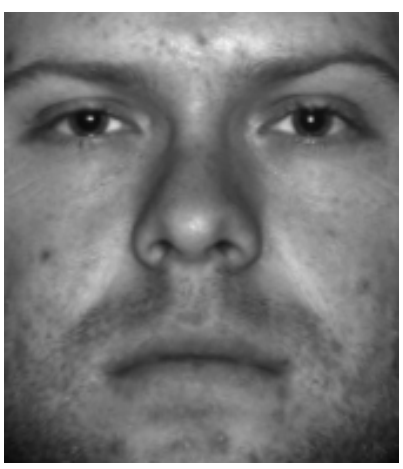

(a) Original image

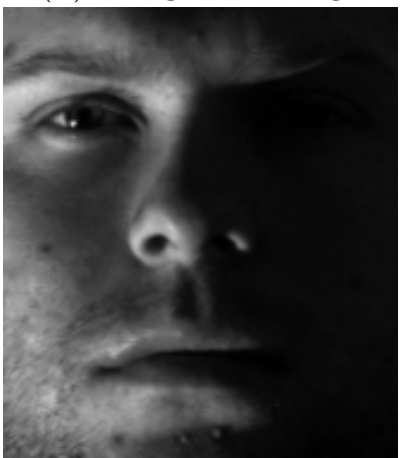

(d) Original image

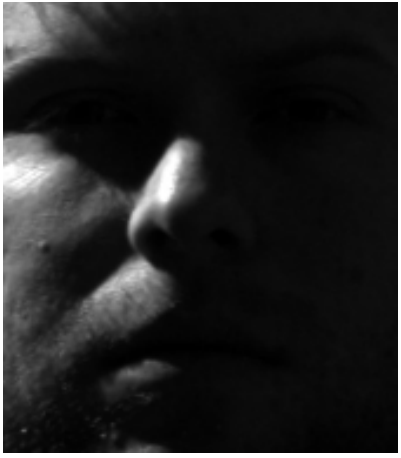

(g) Original image

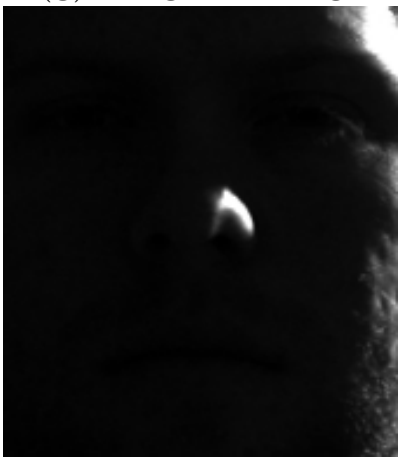

(j) Original image

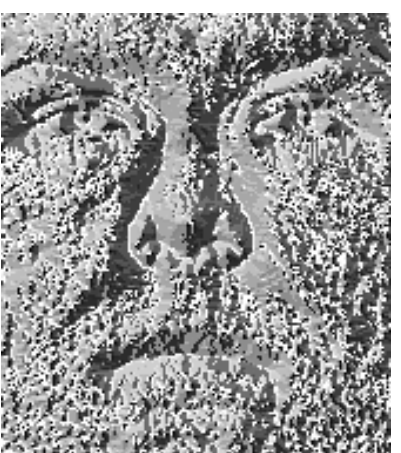

(b) LBP image

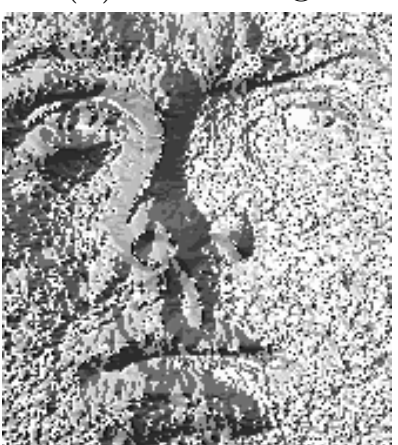

(e) LBP image

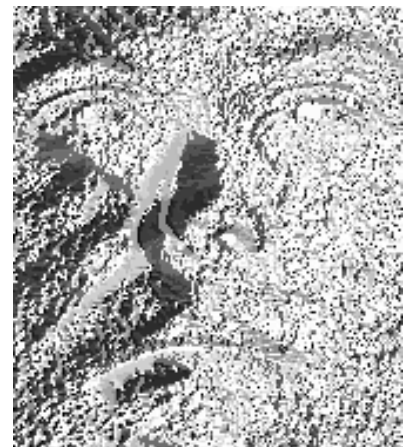

(h) LBP image

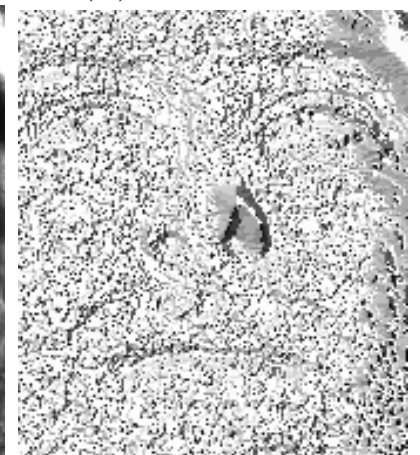

(k) LBP image

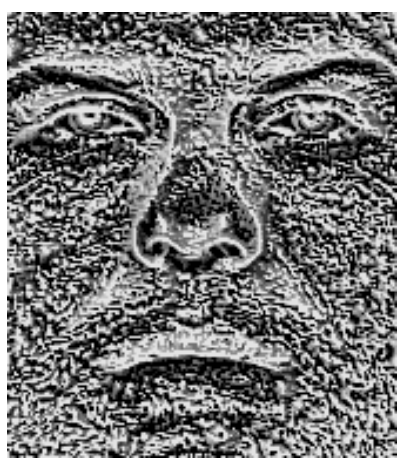

(c) ELBP image

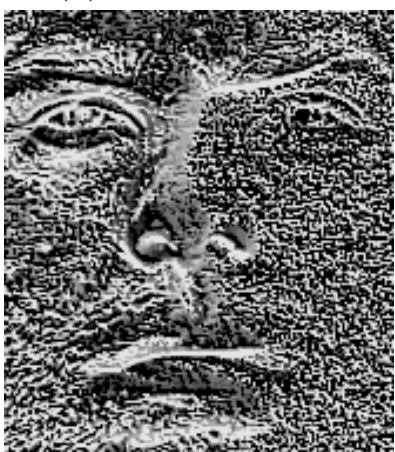

(f) ELBP image

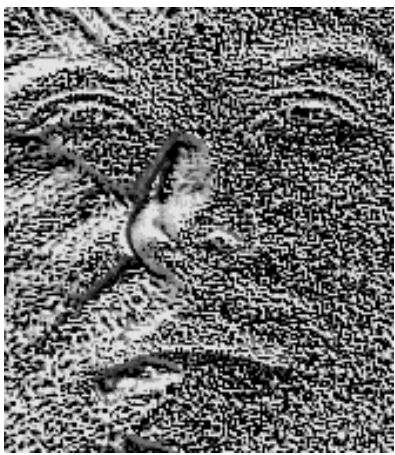

(i) ELBP image

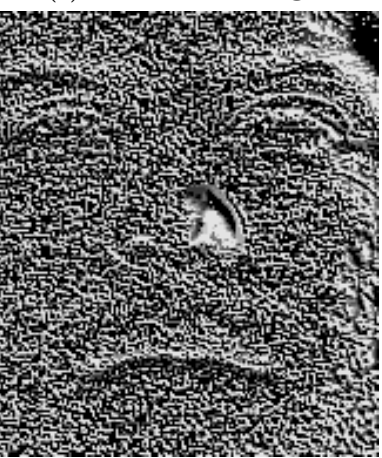

(1) ELBP image

Figure V.6: The comparison between general LBP and ELBP on Extended Yale B database 
into two parts: one with the original Yale B database (10 individuals); the other one with the extended Yale B database (the remaining 28 individuals). Because of the same reason explained in CMU AMP database, ELBP3 was used for obtaining the best result.

\begin{tabular}{|c|c|}
\hline Method & Recognition Rate \\
\hline LBP + A2D-PCA & 97.86 \\
\hline CGP + PCA & 99.29 \\
\hline LDP + A2D-PCA & 99.64 \\
\hline CGP + 2D-PCA & 99.64 \\
\hline Our proposed method & 99.82 \\
\hline
\end{tabular}

Table V.2: The comparison with the results in [59] on Yale B database (10 individuals)

\begin{tabular}{|c|c|}
\hline Method & Recognition Rate \\
\hline LBP + A2D-PCA & 91.02 \\
\hline CGP + PCA & 93.66 \\
\hline LDP + A2D-PCA & 96.05 \\
\hline CGP + 2D-PCA & 97.84 \\
\hline Our proposed method & 98.37 \\
\hline
\end{tabular}

Table V.3: The comparison with the results in [59] on Extended Yale B database (the remaining 28 individuals)

In order to test the "extreme" performance of our proposed method, another experiment is performed: one image (in the neutral lighting condition) of every individual (38 individuals in total) is chosen to be the training image and the others to be the testing images (63 testing images for every individual). Figure V.7 shows the starting point for obtaining the 8-bit code. Table V.4 shows the recognition result of the proposed method. 


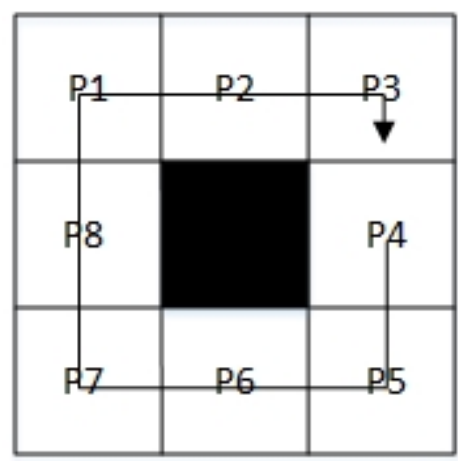

Figure V.7: The order for constructing the 8-bit code: starting point is $P_{4}$ (Extended Yale B database)

\begin{tabular}{|c|c|}
\hline Method & Recognition Rate \\
\hline Our Proposed Method & 89.89 \\
\hline
\end{tabular}

Table V.4: The "extreme" test on Extended Yale B database (38 individuals)

From figure V.6, more detailed information can be extracted after applying ELBP, such as the edge information of the sense organs. Also, the differences of the intensity values caused by the lighting issues are more minimized by ELBP.

\section{V.3 EXPERIMENTS ON PARTIAL OCCLUSION ISSUE}

For testing the recognition results of the images with partial occlusion issue, first, we choose 10 individuals from every database, and manually added occlusions to the images. Only one image per individual is chosen for the training system, and the same image with 6 types of occlusions for the testing system, so that there are 6 testing images in total per individual. Figure V.8 shows the individuals chosen from the four databases. Figure V.8 shows four sets of examples of the added occlusions in four databases (The first image is the training image, and the other 6 images are the testing images for one individual.). The occlusions cover the left eye, right eye, both eyes, nose, horizontal half and vertical half of a face. The recognition results from all 
the databases are $100 \%$. The kind of occlusions may appear on the ID cards, due to the erosion. Our results are promising.

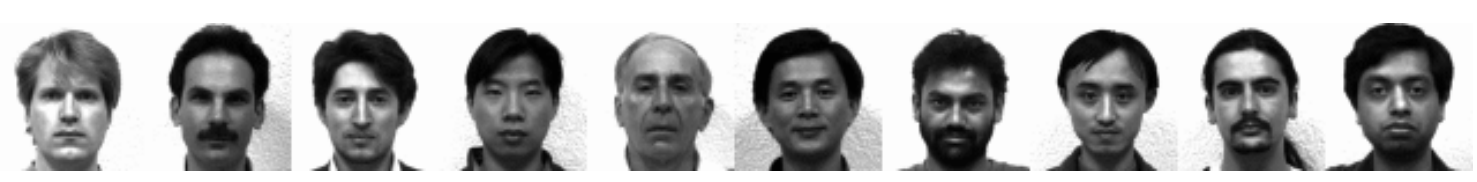

(a) Chosen individuals from Yale database

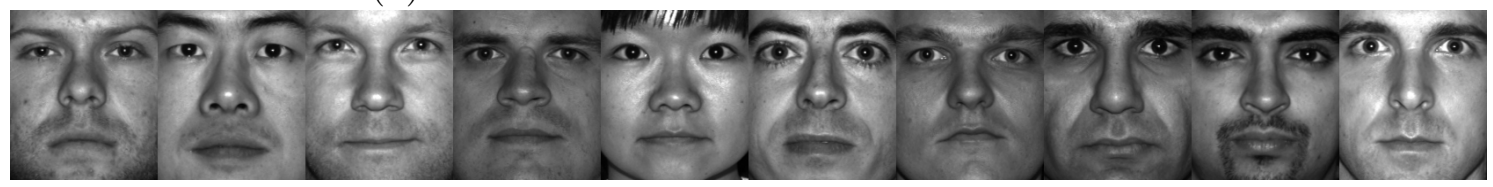

(b) Chosen individuals from Yale B database

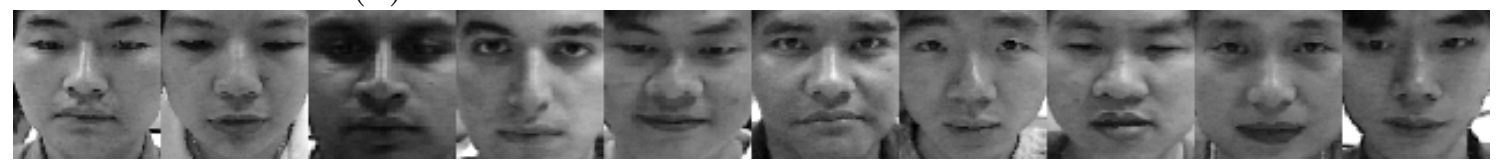

(c) Chosen individuals from CMU AMP Face Expression database

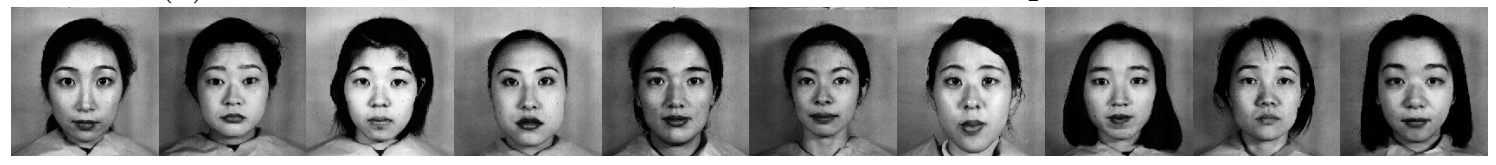

(d) Chosen individuals from JAFFE database

Figure V.8: Individuals chosen from the four databases

Then, the experiments are tested on different testing images (not the same as the training images) on every database. The strategy of adding occlusions is the same as figure V.9. All the individuals are involved in this test. Instead of using all the images except the training images for testing, we choose some of the images which can be exactly classified by the system without any occlusions to be the testing images. For Yale database (15 individuals in total), 6 images per individual used for training (without any occlusions) and 2 other images per individual for testing (with the 6 types of occlusions respectively), so that there are 12 testing images with different types of occlusions per individual, and every type of occlusions has 2 testing images per individual. Every type of occlusions has 30 testing images in total, and the total number of the testing images of all the 15 individuals is 180 . Table V.5 shows the 


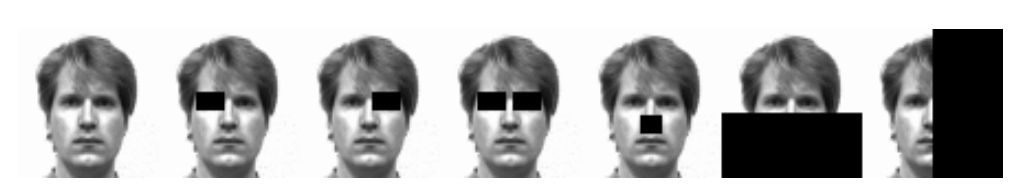

(a) Occlusion examples in Yale database

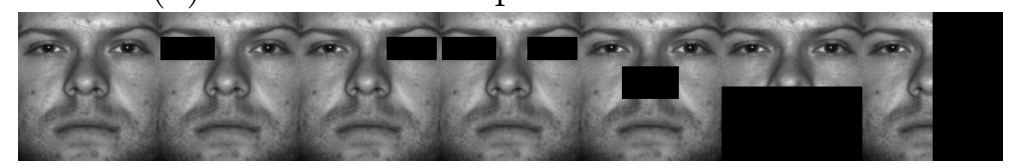

(b) Occlusion examples in Yale B database

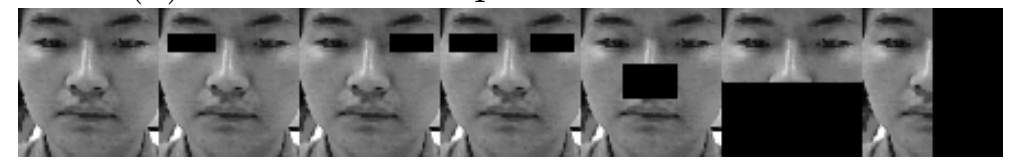

(c) Occlusion examples in CMU AMP Face Expression database

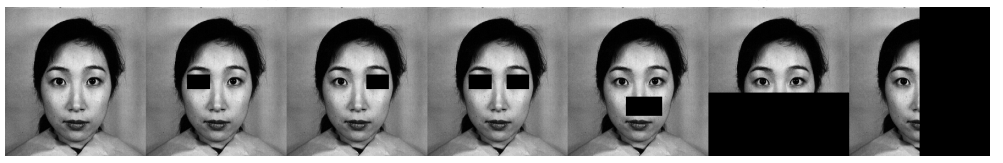

(d) Occlusion examples in JAFFE database

Figure V.9: Occlusion examples in the four databases

recognition results of different types of occlusions.

\begin{tabular}{|c|c|c|}
\hline \multirow{2}{*}{ Occlusion type } & \multicolumn{2}{|c|}{ Recognition result } \\
\cline { 2 - 3 } & WMPCA & Our Proposed Method \\
\hline left eye & 96.67 & 100 \\
\hline right eye & 93.33 & 100 \\
\hline both eyes & 96.67 & 96.67 \\
\hline nose & 93.33 & 100 \\
\hline horizontal half & 33.33 & 93.33 \\
\hline vertical half & 90 & 93.33 \\
\hline average result & 83.89 & 97.22 \\
\hline
\end{tabular}

Table V.5: The recognition results of different types of occlusions on Yale database

From table V.5, for the proposed method, the error of both eyes occlusion is 1 out of 30 , the error of horizontal half occlusion is 2 out of 30 , and the error of vertical half occlusion is 2 out of 30 .

For Yale $\mathrm{B}$ database (38 individuals in total), 7 images per person used for training and 10 other images per person for testing (60 testing images per person 
with 6 types of occlusions). Every type of occlusions has 380 testing images, and the total number of testing images is 2280 . The recognition results of different types of occlusions are shown in table V.6.

\begin{tabular}{|c|c|c|}
\hline \multirow{2}{*}{ Occlusion type } & \multicolumn{2}{|c|}{ Recognition result } \\
\cline { 2 - 3 } & WMPCA & Our Proposed Method \\
\hline left eye & 31.32 & 98.68 \\
\hline right eye & 37.63 & 98.95 \\
\hline both eyes & 26.32 & 95.53 \\
\hline nose & 22.37 & 98.42 \\
\hline horizontal half & 49.74 & 98.68 \\
\hline vertical half & 36.84 & 98.42 \\
\hline average result & 34.04 & 98.11 \\
\hline
\end{tabular}

Table V.6: The recognition results of different types of occlusions on Yale B database

For the proposed method, the error of left eye, right eye, both eyes, horizontal half and vertical half occlusions are 5 out of 380,4 out of 380,17 out of 380,6 out of 380,5 out of 380 , and 6 out of 380 respectively.

From table V.6, the biggest error appears in the "both eyes" occlusion, and the errors of the other types of occlusions are similar, so that the eyes information in Yale B database is very useful for recognition. However, the results are different on CMU AMP and JAFFE databases. For CMU AMP Facial Expression database (13 individuals in total), 10 images per individual used for training and 20 other images per individual used for testing (120 testing images per individual with different types of occlusions). The number of testing images used for every type of occlusions is 260, and the total testing image number is 1560 . Table V.7 shows the recognition results of different occlusions.

For the proposed method, the error of both eyes, horizontal half, and vertical half occlusions are 1 out of 260, 24 out of 260 , and 2 out of 260 respectively. The biggest 


\begin{tabular}{|c|c|c|}
\hline \multirow{2}{*}{ Occlusion type } & \multicolumn{2}{|c|}{ Recognition result } \\
\cline { 2 - 3 } & WMPCA & Our Proposed Method \\
\hline left eye & 95 & 100 \\
\hline right eye & 91.54 & 100 \\
\hline both eyes & 90 & 99.62 \\
\hline nose & 42.69 & 100 \\
\hline horizontal half & 96.15 & 90.77 \\
\hline vertical half & 100 & 99.23 \\
\hline average result & 85.9 & 98.27 \\
\hline
\end{tabular}

Table V.7: The recognition results of different types of occlusions on CMU AMP Facial Expression database

error appears on the horizontal half occlusion, and the other types of occlusions with no or small error. For the horizontal half and vertical half occlusions, the recognition results of WMPCA are a little better than that of the proposed method. This is because the eyes' information of some individuals in CMU AMP database is very similar, and the ELBP space features may minimize the differences between this kind of similarities.

For JAFFE database (10 individuals in total) there are 6 training images and 6 testing images (36 testing images per person with different types of occlusions) per individual. Every type of occlusions has 60 images, and there are 360 testing images in total. Table V.8 shows the recognition results of the 6 types occlusions.

\begin{tabular}{|c|c|c|}
\hline \multirow{2}{*}{ Occlusion type } & \multicolumn{2}{|c|}{ Recognition result } \\
\cline { 2 - 3 } & WMPCA & Our Proposed Method \\
\hline left eye & 96.67 & 100 \\
\hline right eye & 95 & 100 \\
\hline both eyes & 93.33 & 100 \\
\hline nose & 86.67 & 100 \\
\hline horizontal half & 16.67 & 90 \\
\hline vertical half & 88.33 & 90 \\
\hline average result & 87.67 & 98 \\
\hline
\end{tabular}

Table V.8: The recognition results of different types of occlusions on JAFFE database 
For the proposed method, the error of horizontal half and vertical half occlusions are 6 out of 60 and 6 out of 60 respectively. With the results shown above, the most possibility of errors appears in the both eyes, horizontal half and vertical half occlusions, especially in the horizontal half and vertical half occlusions. Too much information is lost when the half of the image is occluded.

For Yale and JAFFE databases, the background information is included, so the results are affected by the background information as well. Compared with the horizontal half occlusion in the four databases, Yale and JAFFE databases have less information than the other two databases, such as the nose information. This causes the recognition rate on horizontal half occlusions of the two databases lower. The horizontal half occlusions for Yale and JAFFE databases are not really the half part of the whole image, because the upper parts of the images of these two databases have some background information, so that the half point of the face is different from the half point of the whole image. Proper boundaries for creating the horizontal half occlusions for Yale and JAFFE databases need to be selected.

Now, one more set of experiments is performed with both eyes occlusion. Figure V.10 shows a set of examples of the occlusions in every database: occlusions are larger than the second set of experiments, as the eyebrows are occluded in these images.
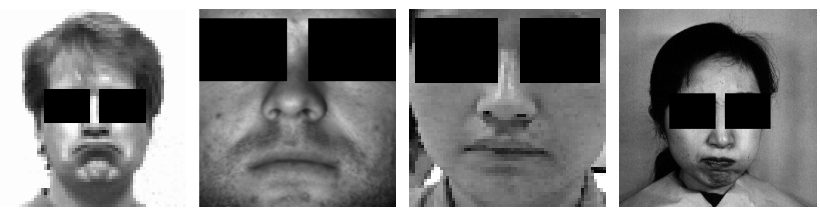

Figure V.10: A set of examples of both eyes and eyebrows occlusions in four databases

The training and testing images are the same as the ones used in the second set of experiments, and the testing images are processed with the both eyes and eyebrows 
occlusions. There are 30, 380, 260 and 60 total testing images for Yale, Yale B, CMU AMP, and JAFFE databases respectively. The recognition results are shown in table V.9.

\begin{tabular}{|c|c|}
\hline Database & Recognition result \\
\hline Yale & 96.67 \\
\hline Yale B & 70.79 \\
\hline CMU AMP & 85.77 \\
\hline JAFFE & 95 \\
\hline
\end{tabular}

Table V.9: The recognition results of both eyes and eyebrows occlusions on four databases

Yale B and CMU AMP databases do not have high recognition results, because these two databases just have the face information. The system makes more errors for classification without other kinds of information, such as the hair information. For Yale B database, even the shape of the face cannot be obtained from the images. For testing the effect of the eyebrows in Yale B and CMU AMP databases, the same size occlusions on the images are made, and the eyebrows are not occluded (Figure V.11). Also, the strategy, the training and testing images are the same as before, but the testing images are added the new both eyes occlusion. Table V.10 shows the recognition results.

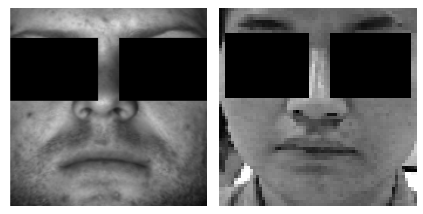

Figure V.11: The new both eyes occlusion (with eyebrows) on Yale B and CMU AMP databases

The increasing of the recognition result of CMU AMP database is obviously. Even though the eyebrows are not occluded in Yale B database, the recognition rate dose not make much improvement. More information needs to be obtained for 


\begin{tabular}{|c|c|}
\hline Database & Recognition result \\
\hline Yale B & 76.32 \\
\hline CMU AMP & 100 \\
\hline
\end{tabular}

Table V.10: The recognition results of the new both eyes occlusion on Yale B and CMU AMP databases

recognizing the individuals on Yale B database. Also, same tests are done with the other two databases, the eyebrows do not have a large influence on the recognition results, because the former results of Yale and JAFFE databases are very good.

\section{V.4 SUMMARY}

With the results from the four databases, the performance of the proposed method shows its effectiveness. For the face expression variation databases, the proposed method is compared with some other methods on Yale database, CMU AMP Facial Expression database, and Japanese Female Facial Expression database. Even though the proposed method may not obtain the best accuracy for every experiment, the efficiency is higher. For the lighting issues, the proposed method yields nearly $90 \%$ accuracy with just one training image in the neutral lighting conditions on Extended Yale B database. Also, the large number of individuals and images are another two challenges for obtaining high accuracy for recognition in Extended Yale B database. For the occlusion issues, the experiment is separated into three parts: one uses the same image for training and testing (add occlusions on the same image); another one uses different training images and testing images; the last one does an extension on the both eyes occlusions. The recognition rate for the first part is amazing. The comparison of the second part shows the capability of the proposed system. The third part gives a different view of the recognition results with both eyes and eyebrows occlusions. 


\section{CHAPTER VI \\ CONCLUSIONS AND FUTURE WORK}

In this thesis, we propose a method that uses the dimensionality reduced ELBP space features for face recognition. The proposed method shows the effectiveness for expression variation, lighting variation and partial occlusion issues. For testing expression variation, three databases are used: Yale database, CMU AMP Facial Expression database, and Japanese Female Facial Expression database. For testing lighting variation, the Extended Yale B database is taken into consideration. For testing partial occlusions, the images are selected from four databases, and occlusions are added manually. The recognition results of the expression variation databases are encouraging. The recognition rates of the lighting variation database show the advantage of the proposed method under poor lighting conditions. Also, the recognition results for the partial occlusion issue are promising.

Comparing with the general LBP + WMPCA (the recognition result shows in figure V.1), the recognition rate of the proposed method is a slight higher. Because of the simplification of the proposed method, the computation cost is still low. Depending on the different size of the cropped parts around the face (whether include the hair, face shape and background information), the proper size of the neighborhood for

computing ELBP descriptor needs to be chosen. In the experiments, Yale database and Japanese Female Facial Expression database use the 5 by 5 neighborhood, and CMU AMP Facial Expression database and Yale B database use the 3 by 3 neigh- 
borhood. Also, the starting point for constructing the 8-bit/16-bit code needs to be considered. This is because the starting point for the comparison between the total absolute distance and total positive distance affects the first group of labels. In the proposed method, the best starting point is obtained by experiments. The starting point affects the accuracy of the result in our experiments. The proposed ELBP generates clearer face images than the general LBP, and the comparisons demonstrate the advantage of the proposed method.

Much effort for improving the recognition rate under different conditions is still needed. The proposed method does not work well in pose variation databases, because the algorithm emphasizes obtaining the new value by the neighbor pixels, and reducing the redundancy caused by the image textures. The pose variation images show different face orientations and positions of key face features (eyes, nose, and mouth) caused by variations of viewing angles. With these kinds of differences, the recognition rate can hardly be improved only with the proposed method. In order to solve the problem, a mutilane approach of trained frameworks at different pose bins is suggested. An appropriate voting strategy need to be used in conjunction with the proposed system. It would lead to a better recognition result in pose variation databases.

For obtaining a better performance, some pre-processing techniques can be added. More testing experiments should be done to find the proper way for improvement. The proposed method can also be applied for facial expression recognition, but a large number of experiments are still needed. The facial expression recognition requires more details from the sense organs. Every sense organ may appear different under a variety of expressions, such as happy, sad, scared, etc. To classify face expressions, more experiments are needed, especially for similar expressions. 


\section{BIBLIOGRAPHY}

[1] Ann Theja Alex, "Local Alignment of Gradient Features for Face Photo and Face Sketch Recognition," M.S.thesis, Dept. Elect. Eng., UD, Dayton, OH, 2012.

[2] Yale Face Database[Online]. Available http://cvc.yale.edu/projects/ yalefaces/yalefaces.html.

[3] Yale B Face Database[Online]. Available http://cvc.yale.edu/projects/ yalefacesB/yalefacesB.html.

[4] X. Liu, T.Chen and B.V.K. Vijaya Kumar, "Face Authentication for Multiple Subjects Using Eigenflow," Pattern Recognition Special issue on Biometric, vol.36, pp.313-328, 2003.

[5] Japanese Female Facial Expression Database[Online]. Available http://www. kasrl.org/jaffe.html.

[6] Facial Recognition System[Online]. Available http://en.wikipedia.org/ wiki/Facial_recognition_system.

[7] Rabia Jafri and Hamid R. Arabnia, "A Survey of Face Recognition Techniques," in Journal of Information Processing Systems (c) 2009 KIPS, doi: 10.3745/JIPS.2009.5.2.041. 
[8] Mark Williams, Better Face Recognition Software[Online]. Available http:// www.itl.nist.gov/iad/News/FaceRecog2.html.

[9] Alexander M. Bronstein, Michael M. Bronstein and Ron Kimmel, Threedimensional Face Recognition*[Online]. Available http://www.cs.technion. ac.il/ ron/PAPERS/BroBroKimIJCV05.pdf.

[10] K. Bonsor and R. Johnson, How Facial Recognition Systems Work[Online]. Available http://electronics.howstuffworks.com/ gadgets/high-tech-gadgets/facial-recognition.htm.

[11] Mike Krause (2002, Jan.14), Is Face Recognition just High-tech Snake Oil?[Online]. Available http://www.enterstageright.com/archive/ articles/0102/0102facerecog.htm.

[12] Birmingham City Centre CCTV Installs Visionics' FaceIt[Online]. Available http://ir.l1id.com/releasedetail. cfm?releaseid=208856.

[13] The University of Milano Bicocca 3D Face Database[Online]. Available http: //www.ivl.disco.unimib.it/umbdb/.

[14] File: Face Detection[Online]. Available http://en.wikipedia.org/wiki/ File:Face_detection.jpg.

[15] L. Sirovich and M. Kirby, "Low-dimensional Procedure for The Characterization of Human Faces," in Journal of the Optical Society of America (c) 1987 Optical Society of America, doi: 10.1364/JOSAA.4.000519.

[16] M. Pietikäinen, A. Hadid , G. Zhao and T. Ahonen, "Local Binary Patterns for Still Images" in Computer Vision Using Local Binary Patterns, New York, pp.13-19, 2011. 
[17] Marko Heikkia, Matti Pietikainen,and Cordelia Schmid, "Description of Interest Regions with Center-Symmetric Local Binary Patterns," Springer-Verlag Berlin Heidelberg, pp.58-69, 2006.

[18] R. Gottumukkal and V.K. Asari, "An Improved Face Recognition Technique based on Modular PCA Approach," Pattern Recognition Letters, vol.25, pp.429436, 2004.

[19] Bernhard Scholkopf, Alexander Smola and Klaus Robert Muller, "Nonlinear Component Analysis as a Kernel Eigenvalue Problem," Nerual Computation,vol.10, pp.1299-1319, 1998.

[20] Bernhard Scholkopf, Alexander Smola and Klaus Robert Muller, "Kernel Principal Component Analysis", in Advances in Kernel Methods - Support Vector Learning, Cambridge, MA: MIT Press, 1998.

[21] Zizhu Fan, Ergen Liu, and Baogen Xu, "Weighted Principal Component Analysis," in Artificial Intelligence and Computational Intelligence, vol.7004, pp.569$574,2011$.

[22] Chengjun Liu. "Gabor-based Kernel PCA with Fractional Power Polynomial Models for Face Recognition," IEEE Transactions on Pattern Analysis and Machine Intelligence, vol.26, pp.572-581, 2004.

[23] Jian Yang, David Zhang, Senior Member, IEEE, Alejandro F. Frangi, and Jingyu Yang, "Two-dimensional PCA: A New Approach to Appearance-based Face Representation and Recognition," IEEE Transactions on Pattern Analysis and Machine Intelligence, vol.26, pp.131-137, 2004.

[24] L10: Linear discriminants analysis[Online]. Available http://research.cs. 
tamu.edu/prism/lectures/pr/pr_110.pdf.

[25] S. Har-Peled, D. Roth, D. Zimak, "Constraint Classification for Multiclass Classification and Ranking," in In Proceedings of the 16th Annual Conference on Neural Information Processing Systems, Cambridge, MA: MIT Press, pp.785$792,2003$.

[26] Niklas Lavesson, "An Analysis of Approaches to Evaluate Learning Algorithms and Classifiers" in Evaluation and Analysis of the Supervised Learning Algorithm and Classifier, Sweden: Blekinge Institute of Technology, pp.13, 2006.

[27] Peter Dayan, "Unsupervised Learning" in The MIT Encylopedia of the Cognitive Sciences, Cambridge, MA: MIT Press, 2001.

[28] Xiaojin Zhu, "Semi-Supervised Learning Literature Survey"[Online]. Available http://www.Ioni.ucla.edu/ ztu/courses/2013_CS_spring/reading/ ssl_survey.pdf.

[29] Elena Deza and Michel Marie Deza, Encyclopedia of Distances,2nd ed., 2013.

[30] P. C. Mahalanobis, "On the Generalised Distance in Statistics," in Proceedings of the National Institute of Sciences of India 2 (1), vol.2, pp.49-55, 1936.

[31] R. De Maesschalck, D. Jouan-Rimbaud and D. L. Massart, The Mahalanobis distance, Chemometrics and Intelligent Laboratory Systems, pp.1-18, 2000.

[32] Artificial Neural Network - Perceptron[Online]. Available http://chem-eng. utoronto.ca/ datamining/dmc/artificial_neural_network_bkp.htm.

[33] Leonardo Noriega, Multilayer Perceptron Tutorial[Online]. Available http://www.cs.sun.ac.za/ kroon/courses/machine_learning/lecture5/ mlp.pdf. 
[34] Boaz Lerner, Hugo Guterman, Mayer Aladjem and Itshak Dinstein, "A Comparative Study of Neural Network based Feature Extraction Paradigms," in Pattern Recognition Letters, vol.20, pp.7-14, 1999.

[35] Linda G. Shapiro and George C. Stockman, Computer Vision, 1st ed.,Upper Saddle River, NJ:Prentice Hall, 2001.

[36] Mona Sharma,Markos Markou and Sameer Singh, Evaluation of Texture Methods for Image Analysis[Online]. Available http://www.eng.iastate.edu/ ee528/Papers/texturecomparison.pdf.

[37] J.F. Haddon and J.F. Boyce, "Co-occurrence Matrices for Image Analysis," IEEE Electronics and Communications Engineering Journal, vol.5, pp.71-83, 1993.

[38] K. Laws, "Textured Image Segmentation," Ph.D. Dissertation, USC, Los Angeles, CA, 1980.

[39] DC. He and L. Wang, "Texture Unit, Texture Spectrum, and Texture Analysis," Geoscience and Remote Sensing, vol.28, pp.509 - 512, 1990.

[40] T. Ojala, M. Pietikäinen, and D. Harwood, "Performance Evaluation of Texture Measures with Classification based on Kullback Discrimination of Distributions," Pattern Recognition, vol.1, pp.582 - 585, 1994.

[41] M. Pietikäinen, T. Ojala and Z. Xu, "Rotation-invariant Texture Classification Using Feature Distributions," Pattern Recognition, vol.33, pp.43-52, 2000.

[42] Lindsay I. Smith, A Tutorial on Principal Components Analysis[Online]. Available http://www.ce.yildiz.edu.tr/personal/songul/file/1097/ principal_components.pdf. 
[43] K. Pearson, "On Lines and Planes of Closest Fit to Systems of Points in Space," Philosophical Magazine, vol.2, pp.559-572, 1901.

[44] Principal Component Analysis[Online]. Available http://www.fon.hum.uva. nl/praat/manual/Principal_component_analysis.html.

[45] T. Ojala, M. Pietikäinen and D. Harwood, "A Comparative Study of Texture Measures with Classification based on Feature Distributions," Pattern Recognition vol.29, pp.51-59, 1996.

[46] L. Wang and D. C. He, "Texture Classification using Texture Spectrum," Pattern Recognition, vol.23, pp.905-910, 1990.

[47] T. Ojala, M. Pietikäinen and T. Mäenpää, "Multiresolution Gray-scale and Rotation Invariant Texture Classification With Local Binary Patterns," Pattern Analysis and Machine Intelligence, vol.24, pp.971-987, 2002.

[48] Bilinear Inteporlation[Online]. Available http://en.wikipedia.org/wiki/ Bilinear_interpolation.

[49] Principal Component Analysis[Online]. Available http://support.sas.com/ publishing/pubcat/chaps/55129.pdf.

[50] Mohammad Moinul Islam, Vijayan K. Asari, Mohammed Nazrul Islam, and Mohammad A. Karim, "Modified Local Binary Pattern (MLBP) for Robust Face Recognition," Proceedings of the International Conference on Neural Computation Theory and Applications, Paris, France, pp.147-152, 2011.

[51] A. Pavan Kumar, Sukhendu Das and V. Kamakoti, "Face Recognition Using Weighted Modular Principle Component Analysis", in Neural Information Processing, vol.3316, pp.362-367, 2004. 
[52] Wei Xia, Shouyi Yin and Peng Ouyang, "A High Precision Feature Based on LBP and Gabor Theory for Face Recognition," Sensors, vol.13, pp.4499-4513, doi:10.3390/s130404499.

[53] A.M. Martinez and A.C. Kak. "PCA versus LDA," Pattern Analysis and Machine Intelligence, vol.23, pp.228-233, 2001.

[54] H. Qin, L. Qin, L. Xue, Y. Li. "A kernel Gabor-based weighted region covariance matrix for face recognition," Sensors, vol.12, pp.992-993, 2012.

[55] J. Wright, A. Y. Yang, A. Ganesh, S. S. Sastry, and Y. Ma., "Robust Face Recognition via Sparse Representation," Pattern Analysis and Machine Intelligence, vol.31, pp.210-227, 2009.

[56] P. Nagesh and B. Li, "A Compressive Sensing Approach for Expressioninvariant Face Recognition," Computer Vision and Pattern Recognition, pp.1518-1525, 2009.

[57] Ming-Jung Seow, Ann Theja Alex and Vijayan K. Asari, "Learning Embedded Lines of Attraction by Self Organization for Pose and Expression Invariant Face Recognition," Opt. Eng., vol.51, doi :10.1117/1.OE.51.10.107201, 2012.

[58] M. Turk and A. Pentland, "Eigenfaces for Recognition," Journal of Cognitive Neuroscience, vol.3, pp.71-86, 1991.

[59] K. Dong-Ju, L. Sang-Heon, S. Myoung-Kyu, K. Byungmin and K. Hyunduk, A Novel Image Preprocessing Technique Based on Binary Patterns[Online]. Available http://onlinepresent.org/proceedings/vo14_2012/34.pdf. 